


\title{
Contractile Performance of Striated Muscle
}

\author{
By \\ K.A.P. Edman \\ Department of Experimental Medical Science, Biomedical Centre \\ University of Lund, S-221 84 Lund, Sweden
}




\begin{abstract}
The single muscle fibre preparation provides an excellent tool for studying the mechanical behaviour of the contractile system at sarcomere level. The present article gives an overview of studies based on intact single fibres from frog and mouse skeletal muscle. The following aspects of muscle function are treated: 1 . The length-tension relationship. 2 . The biphasic force-velocity relationship. 3. The maximum speed of shortening, its independence of sarcomere length and degree of activation. 4. Force enhancement during stretch, its relation to sarcomere length and myofilament lattice width. 5. Residual force enhancement after stretch. 6. Force reduction after loaded shortening. 7. Deactivation by active shortening. 8 . Differences in kinetic properties along individual muscle fibres.
\end{abstract}




\section{Introduction}

A study published in Nature 1939 by Engelhardt \& Ljubimova can be said to form the beginning of a new era in muscle physiology. It was demonstrated in this article that “myosin”, known at the time to be one of the principal components of striated muscle (Bailey, 1937), had the ability to split the high-energy phosphate compound adenosine triphosphate (ATP). The Engelhardt-Ljubimova discovery inspired the Hungarian biochemist Albert Szent-Györgyi to further elucidate the "myosin"-ATP reaction and its relation to force development and movement of muscle. In the years to follow (1940-1946), while Europe was ravaged by war, Szent-Györgyi and his school made the most fundamental discoveries ever in muscle physiology. What was known as "myosin" at the time was found to be a complex of two proteins: the true myosin (initially named myosin A) and a newly identified protein, named actin. The discovery of actin was made by F.B. Straub (1942), a member of SzentGyörgyi’s team. The actin-myosin complex (actomyosin) could be extracted from muscle tissue in a high-salt solution and finally shaped into threads at physiological ionic strength. The threads so produced were shown to contract heavily on addition of ATP, a phenomenon that Albert Szent-Györgyi in later years described as the most impressive phenomenon he had experienced in his scientific career. Significant contributions in the early studies of the contractile machinery also came from the laboratory of H.H. Weber (1952). It was demonstrated by Weber and co-workers (Weber and Portzehl 1952) that ATP has two important actions on reconstituted actomyosin fibres: it induces contraction and at the same time makes the fibres plastic, the latter effect being demonstrable by blocking the myosin ATPase activity by means of an SH-group reagent. It should be noted at this point that actomyosin lacking the regulatory system, the troponin-tropomyosin complex, does not require calcium to be contracted by ATP. 
Near the end of the war, before the borders of Hungary were effectively closed for immigration, Albert Szent-Györgyi left Budapest to spend half a year at the Karolinska Institute in Stockholm, Sweden. During this time he was invited to republish a review article of his seminal work on muscle in Acta Physiologica Scandinavica (1944). Before proceeding to the US Albert Szent-Györgyi arranged for two of his previous pupils in Budapest to come to work at Uppsala University. One of them, T. Erdös, had previously demonstrated, at the suggestion of Szent-Györgyi, that the state of rigor in muscle is due to depletion of ATP, i.e. loss of the plasticizing action of ATP. My own interest in muscle function came in at this point. as I happened to be a research student at the Department of Pharmacology at Uppsala University where one of Szent-Györgyi’s previous pupils (A. Czapó) came to work. My first papers (Edman, 1950, 1951, 1953) describe the effects of cardiac glycosides on the ATPase activity of cardiac myosin. In parallel with this work and together with my mentor E.H. Bárány, an electronic technique was developed for measuring the time course of dissociation of actomyosin in solution after addition of ATP. Our main interest in this project was to establish how calcium and magnesium and variations in the electrolyte composition of the medium might affect the rate of dissociation of actomyosin in response to ATP (Bárány et al. 1951a, 1951b, 1952). This was followed by detailed studies of the relaxing effects of zinc in glycerinated (skinned) muscle fibres (e.g. Edman, 1959a, 1959b). The essential role of the sarcoplasmic reticulum in the relaxation process was unknown at the time, and the possible role of zinc as a relaxing agent became a most intriguing possibility after observing that there is as much zinc in muscle as there is calcium. My subsequent work on muscle has mainly been performed on intact preparations of smooth muscle (Edman \& Schild, 1962, 1963), myocardium (Edman \& Nilsson, 1968, 1972) and intact skeletal muscle fibres. The latter preparations have enabled studies of the contractile machinery at sarcomere level using 
various mechanical and optical techniques. In the following account some basic properties of the contractile system will be described as elucidated in the single fibre preparation.

\section{The length-tension relationship in striated muscle}

It was demonstrated in early studies on isolated frog muscle by M. Blix (professor of Physiology at the University of Lund; Blix, 1895) that the force producing capability of skeletal muscle is critically dependent on the degree of extension of the muscle. Blix demonstrated that, after account had been made for passive tension, there was a bell-shaped relationship between active force and muscle length and that maximum force was produced near the length assumed by the muscle at rest in the body. It soon became clear that the length-tension relationship contains information of great relevance to our understanding of the contractile process, and the observations presented by Blix (1894) were confirmed and extended in several subsequent studies on both whole muscle (Evans \& Hill, 1914) and single muscle fibres of the frog (Ramsey \& Street, 1940) and on partially isolated voluntary muscles of human subjects (Ralston et al., 1947). With the presentation of the sliding-filament hypothesis of muscle contraction (Huxley HE \& Hanson, 1954; Huxley AF \& Niedergerke, 1954) it became vital to relate force production and shortening velocity to sarcomere length, i.e. to relate the mechanical performance of the muscle to the state of overlap between the thick (myosin) and thin (actin) filaments that make up the sarcomeres. The first two studies with this particular aim, both carried out on intact muscle fibres (M. semitendinosus) of the frog, were published in the same journal by Gordon et al. (1966) and Edman (1966). Both studies demonstrated that maximum tetanic force increased with increasing filament overlap reaching a maximum at a sarcomere length where the thin filaments would reach the central, inert, zone of the thick filaments. The paper by Edman (1966) was specifically focussed on the question whether the contractile state at a given sarcomere length might depended on how 
this length is being reached, by passive movement at rest or by active shortening from a prestretched position. It was found that, provided the fibre is allowed to shorten against a light load, the starting length does not affect the force producing capability at the end of the movement. That is, the isometric force was found to be determined by the actual sarcomere length only, in full accordance with the sliding filament hypothesis.

The studies reported by Gordon et al. (1966) and Edman (1966) were both carried out on fairly long fibre preparations in which intrinsic, non-uniform sarcomere movements might affect force recording. A renewed study of the length-tension relationship was therefore carried out (Edman \& Reggiani, 1987), again on isolated frog muscle fibres, using a technique by which isometric force and shortening velocity could be performed on short (0.5$0.7 \mathrm{~mm}$ ) marked segments along the length of the fibre. By this technique, described in detail by Edman \& Reggiani (1984), a limited population of sarcomeres (circa 150-300 sarcomeres in series) could be held at constant overall length during tetanic stimulation or, alternatively, be allowed to shorten actively against a constant, preset load. The length-tension relationship depicted by the latter technique (Fig. 1) differs in certain respects from the polygonal lengthtension curve proposed by Gordon et al. (1966). The new data showed that the length-tension relationship has a smoother shape. It exhibits no distinct plateau between 2.0 and $2.2 \mu \mathrm{m}$ sarcomere length, and the descending limb can be seen to have a slightly sigmoid shape with the main portion extrapolating to a distinctly shorter sarcomere length $(3.49 \mu \mathrm{m})$ than that (3.65 $\mu \mathrm{m}$ ) postulated by Gordon et al. (1966). As demonstrated by Edman \& Reggiani (1987) the length-tension relationship, even in its revised form, is fully explainable in terms of the sliding-filament hypothesis. The results are consistent with the view that: 1 . there is a substantial variability (S.D. $0.2 \mu \mathrm{m}$ ) in the degree of overlap between the thick (A) and thin (I) filaments and 2. the average functional length of the A and I filaments is 1.55 and 1.94 $\mu \mathrm{m}$, respectively, in frog striated muscle. These data agree closely with the revised values of 
the A and I filament lengths (1.55 and 1.92-1.96 $\mu \mathrm{m}$, respectively) that were presented by Page (1968) and Huxley (1973). Several factors may contribute to the variability in filament overlap predicted by the length-tension curve: i. non-uniformity in length of the thin
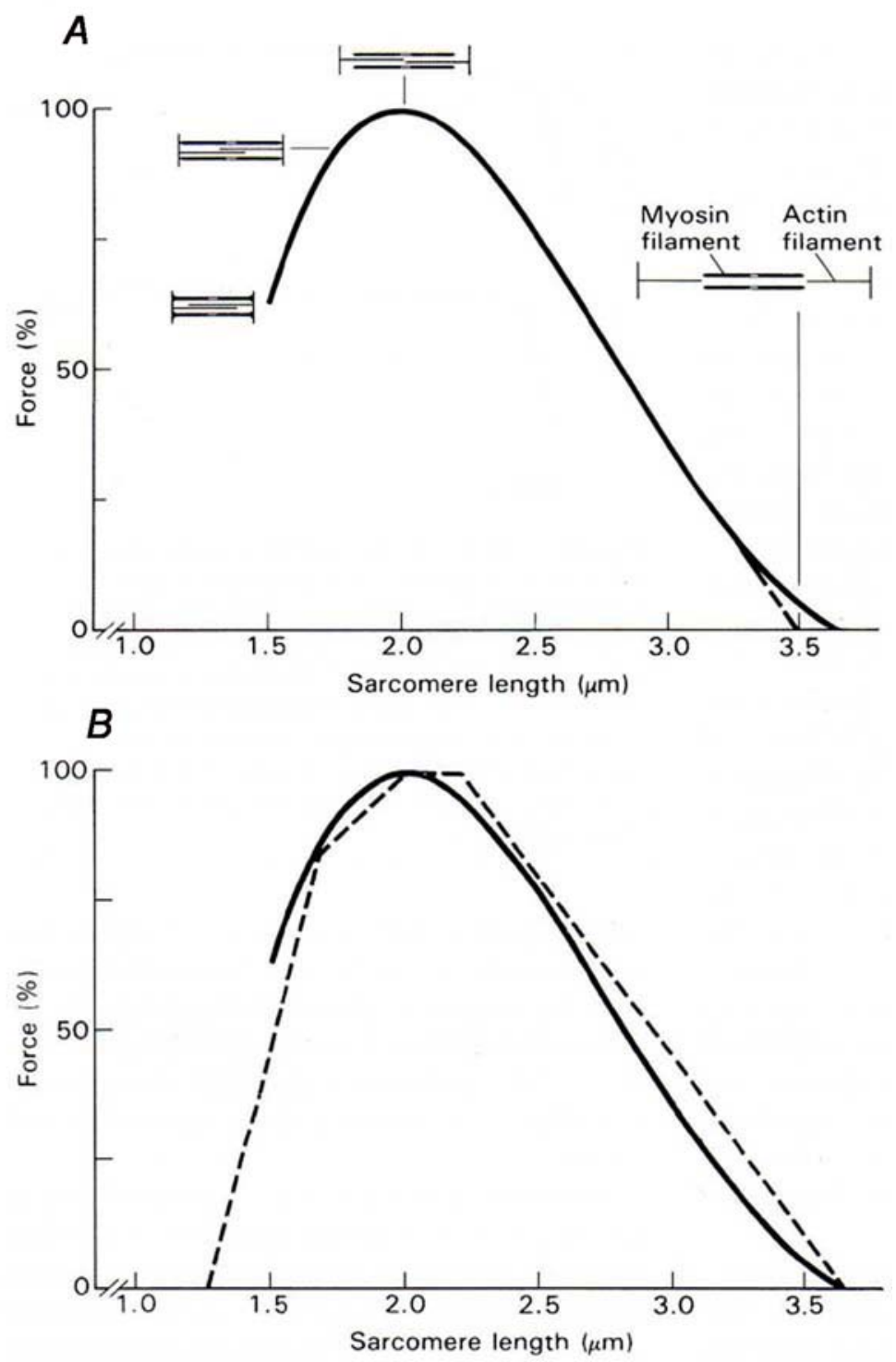

Fig. 1. A. Variation of maximum tetanic force with sarcomere length. Curve from Edman \& Reggiani (1987) based on measurements from short, length-controlled segments of single muscle fibres. The dashed line shows extrapolation to abscissa of the steep portion of the length-tension relation. The intersection of the dashed line with the abscissa marks the sarcomere length at which the majority of the A- and I-filaments are in end-to-end position. Insets show degree of filament overlap at four different sarcomere lengths.

B. The length-tension relationship shown in A (full line) compared with the polygonal length-tension curve (dashed line) described by Gordon et al. (1966).

filaments, ii. longitudinal misalignment (staggering) of the thick and thin filaments within the A and I bands of the sarcomere and iii. differences in sarcomere length (mean M-Z line 
spacing) among individual myofibrils within a given cross-section of the fibre. It is essential to point out that a randomized variation of filament overlap is entirely consistent with the finding that the sarcomere pattern remains constant during length-clamp recording of a short fibre segment. This has to do with the fact that elastic structures connecting adjacent myofibrils across the fibre will be strained and will support those sarcomeres, or sarcomere halves, that have less capacity to develop force than has a neighbouring region (see further Edman \& Tsuchiya, 1996).

The first study of the length-tension relationship performed on mammalian intact single muscle fibres was recently presented (Edman, 2005). The experimental approach was essentially the same as that employed in the earlier frog fibre experiments, i.e. tetanic force was recorded from a short (ca. $0.3 \mathrm{~mm}$ ) marked segment that was held at constant length during stimulation. The fibres dissected from the flexor digitorum brevis muscle of the mouse

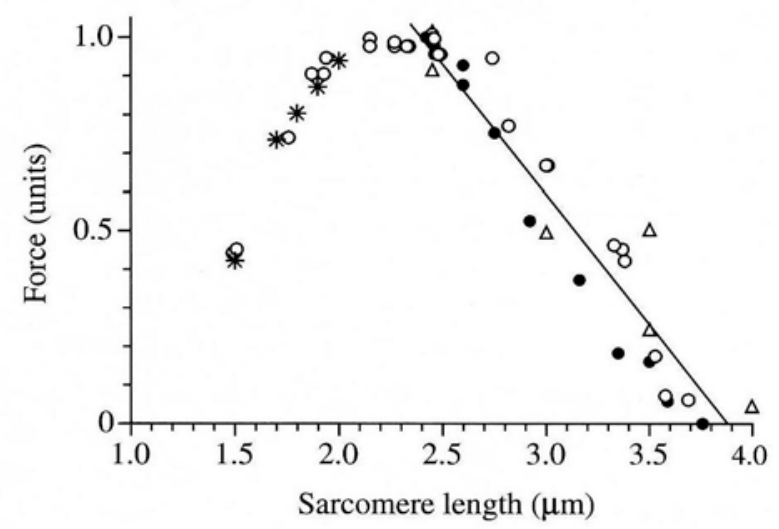

Fig. 2. Tetanic force versus sarcomere length in mouse single muscle fibres. Force is expressed in units of the tetanic force recorded near $2.4 \mu \mathrm{m}$ sarcomere length. The straight line is linear regression of force upon sarcomere length for values between 2.35 and $4.00 \mu \mathrm{m}$ sarcomere length. The asterisks along the ascending limb are calculated values based on the assumption that force is reduced below optimum length in proportion to the occurrence of double filament overlap and, at more extreme degrees of shortening, to compression of the thick filaments against the Z disks. For further details, see Edman (2005).

were merely $25-40 \mu \mathrm{m}$ wide and $0.5 .-0.7 \mathrm{~mm}$ in length between the tendon insertions. As illustrated in Fig. 2, the length-tension relationship has a shape that, similar to the situation in 
amphibian muscle fibres, can be fitted well with the sliding-filament hypothesis. The slope of the descending limb predicts that the average length of the thin filaments is $1.10 \mu \mathrm{m}$ and the length of the thick filaments is $3.88 \mu \mathrm{m}$, both values being in excellent agreement with recent electron microscopical measurements of the two filaments in rabbit psoas muscle (Sosa et al., 1994).

\section{The force-velocity relationship. Maximum velocity of shortening}

In contrast to a simple elastic body active muscle has the remarkable property to adjust its force to precisely match the load that is experienced during shortening. It does so by continuously adjusting the speed at which it moves. Thus, when the load is small, the force produced by the contractile system is made correspondingly small by increasing the speed of shortening appropriately. Conversely, when the muscle is heavily loaded, the active force is raised to an equivalent level by reducing the shortening velocity sufficiently. Fenn \& March (1935) were first to formulate the inverse relationship between load and velocity of shortening. Hill (1938) further characterized the force-velocity relationship and described it in terms of a rectangular hyperbola. Hill also pointed out the important role that the forcevelocity relationship is likely to have for understanding the basic mechanisms of muscle contraction.

A detailed study on single muscle fibres (Edman, Mulieri \& Scubon-Mulieri, 1976) revealed that the force-velocity relationship deviates from Hill's hyperbolic equation in the high-force range. Improved recording techniques, including measurements from short lengthcontrolled segments of intact fibres, made it possible to characterize this phenomenon in considerable 



Fig. 3. Force-velocity relationship based on measurement in a single muscle fibre. Velocity of shortening recorded in the fibre as a whole $(A)$ and in a short (ca. $0.6 \mathrm{~mm}$ ) segment of the same fibre $(B)$. Note difference in curvature of force-velocity relation on either side of a break point near $0.8 P_{0}$. Continuous lines: Hill's hyperbolic equation fitted to data points above and below the break point. Insets: semilogarithmic plots of the force-velocity data given in the main diagrams. Lines in the insets, linear regressions of velocity upon force on either side of the break point. Sarcomere length: $2.10 \mu \mathrm{m}$. Temperature: $2.2{ }^{\circ} \mathrm{C}$.

$C$, force-velocity data (from whole fibre) derived in the high-force range plotted in diagram in which the ordinate has been placed at $0.80 P_{0}$. Hyperbola fitted by least-squares method. From Edman (1988).

detail (Edman, 1988). As illustrated in Fig. 3 the force-velocity relationship exhibits two distinct curvatures, both with an upward concavity on either side of a breakpoint near $80 \%$ of the isometric force $\left(\mathrm{P}_{0}\right)$. The breakpoint is most clearly illustrated by plotting the force- 
velocity data in a semilogarithmic diagram as shown in the insets of Fig. 3. The biphasic shape of the force-velocity relationship, with a breakpoint near $0.8 \mathrm{P}_{0}$, also appears in a similar way in mammalian muscle fibres (Edman, 2005; Devrome \& MacIntosh, 2007) and in isolated muscle spindles of the frog (Edman et al., 2002). It is worth

pointing out that the high-force deviation of the force-velocity curve has been shown to exist also in skinned muscle fibres and to be unrelated to the state of activation of the contractile system (Lou \& Sun, 1993). The biphasic shape of the force-velocity relation thus seems to reflect a basic feature of the sliding-filament process. The following empirical equation provides an excellent fit to the experimental data (Fig. 4):

$$
V=\frac{\left(P_{0}^{*}-P\right) b}{P+a}\left(1-\frac{1}{1+e^{-k_{1}\left(P-k_{2} P_{0}\right)}}\right)
$$

$V$ denotes the velocity of shortening, $P$ the load on the muscle fibre and $P_{0}$ is the measured isometric force. The first term of the equation expresses the force-velocity relation below $0.8 \mathrm{P}_{0}$ and represents a rectangular hyperbola in the form described by Hill (1938). $\mathrm{P}_{0}{ }^{*}$ is the isometric force predicted from this hyperbola and $a$ and $b$ are constants with dimensions of force and velocity, respectively. The second term within parenthesis (referred to as the 'correction term'; Edman, 1988) reduces V at high loads to fit the distinct upward-concave curvature at loads greater than approximately $0.8 P_{0}$. The constant $k_{1}$ in the correction term has the dimension of 1 /force, whereas $k_{2}$ is dimensionless. $k_{1}$ determines the steepness of the high-force curvature and $k_{2}$ the relative force at which the correction term reaches its half value. The two constants are useful parameters when comparing data from different studies. 


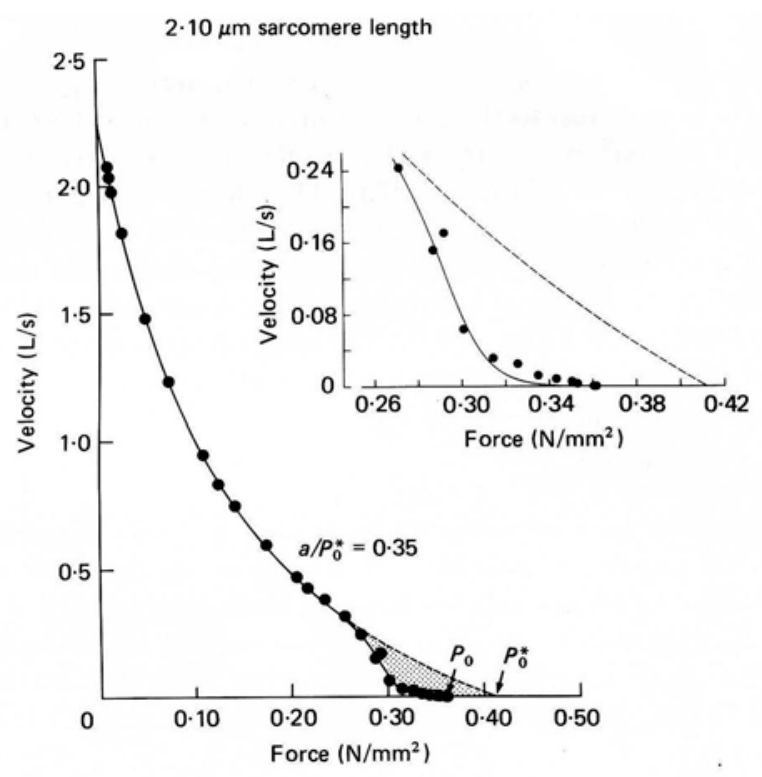

Fig. 4. Equation 1 fitted to force-velocity data derived from a single muscle fibre. Principle of curve fitting: The first term in eqn. 1 (Hill's hyperbolic equation) was initially fitted, by least-squares method, to data truncated at $0.78 P_{0}$ in this way deriving the numerical values of constants $a$ and $b$. Constants $k_{1}$ and $k_{2}$ were thereafter determined by fitting of eqn. 1 to all data points, i.e. including loads greater than $0.78 P_{0}$. Inset: detail of highforce portion of the force-velocity relation shown in main diagram. Continuous line, fit of eqn. 1 . Dashed line, extrapolation of hyperbola derived from data below $0.78 P_{0}$. From Edman (1988)

Several attempts have been made to simulate the characteristic change of the force-velocity relation in the high-force range using different cross-bridge models (Mitsui \& Chiba, 1996; Edman et al. 1997; Duke, 1999; Nielsen, 2003). The model presented by Edman, Caputo \& Månsson (1997) simulates the experimental force-velocity and stiffness-velocity relationships exceedingly well. According to this model there is a Gaussean position-dependence of the attachment rate constant along the thin filament. This will provide a region early during the power stroke where the cross-bridge attachment is slow. This feature of the model leads to a marked decrease in the number of pulling cross-bridges during shortening in the high-force range, and to a lower force output per bridge. Taken together, these changes account for the upwards-concave shape of the force-velocity relation at high loads (0.8 $\left.P_{0}-P_{0}\right)$ according to the model.

There is reason to believe that the maximum speed of shortening $\left(\mathrm{V}_{\max }\right)$, which is attained when the muscle is free to shorten at zero load, can be presumed to express the maximum 
cycling rate of the myosin cross-bridges. Supporting this view $\mathrm{V}_{\max }$ has been found to correlate well with the maximum rate of splitting of ATP within the contractile system. This was first indicated in studies of whole muscles (Bárány, 1967), and later in a more quantitative way (Edman, Reggiani, Schiaffino \& te Kronnie, 1988), by correlating $\mathrm{V}_{\max }$ and myofibrillar ATPase activity in isolated single muscle fibres (Fig. 5).

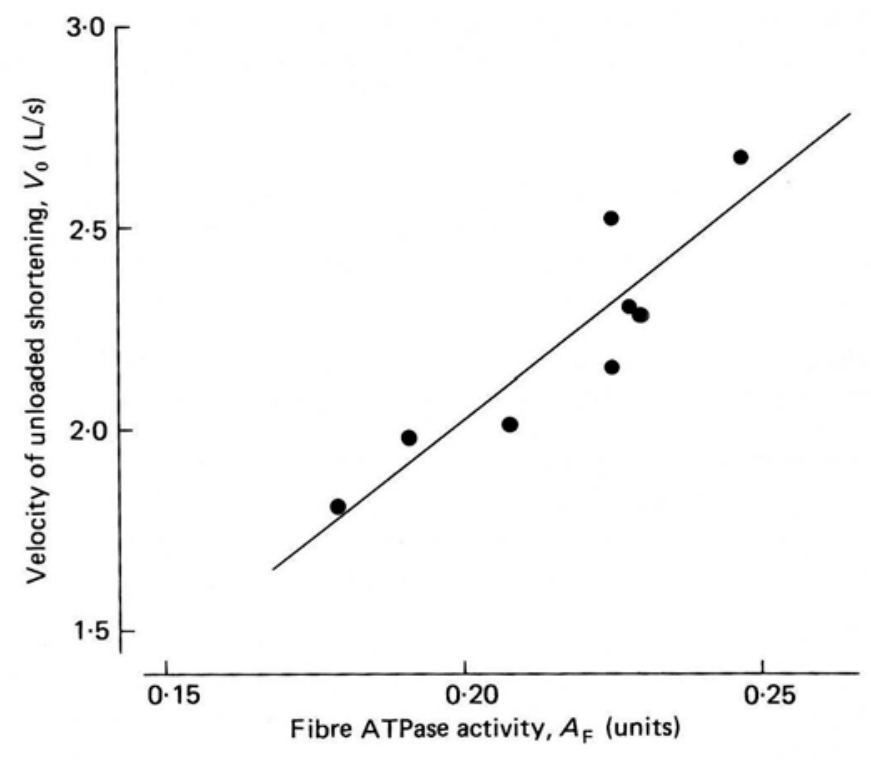

Fig. 5. Relationship between velocity of unloaded shortening $\left(V_{0}\right.$, Lengths/s) and myofibrillar ATPase activity ( $A_{\mathrm{F}}$, units) in eight anterior tibialis muscle fibres from frogs. Line: least-squares regression of $V_{0}$ upon $A_{\mathrm{F}}$. From Edman et al. (1988).

A critical test of the sliding-filament model is establishing whether $V_{\max }$ varies with the number of cross-bridges that interact with the thin filament. If $\mathrm{V}_{\max }$ represents the maximum speed at which the cross-bridges are able to cycle, $\mathrm{V}_{\max }$ may be presumed to be independent of the actual number of interacting bridges. That is, maximum speed of shortening would be expected to stay constant at different degrees of overlap between the thick and thin filaments and, also, at different degrees of activation of the contractile system. The results illustrated in 



Fig. 6. A. Maximum velocity of shortening $\left(\mathrm{V}_{\max }\right.$, right ordinate) $)$ measured at various sarcomere lengths in three different fibres (indicated by different symbols). $\mathrm{V}_{\max }$ can be seen to remain very nearly constant as sarcomere length is changed. The dashed line shows, for comparison, the variation in isometric force within the range of sarcomere lengths considered (1.6 - $2.8 \mu \mathrm{m})$. Replotted data from Fig. 5 of Edman (1979).

$B$. Maximum speed of shortening $\left(V_{\max }\right)$ recorded at different degrees of activation of single muscle fibres. Open circle, $V_{\max }$ during tetanus, i.e. at full activation in the respective fibre. Filled circles, measurements of $V_{\max }$ during twitch contractions representing various degrees of activation as indicated on abscissa. $V_{\max }$ can be seen to remain quite constant as activation is changed. (Plotting of data from Table 1 of Edman, 1979).

Fig. 6) clearly demonstrate that these predictions hold true. Fig. 6A shows that, over a wide range of sarcomere lengths $(1.6-2.7 \mu \mathrm{m})$, the maximum speed of shortening remains very nearly constant in the individual muscle fibre while, over the same range of sarcomere lengths, there is a great variation in the fibre's capacity to produce force. Thus, in contrast to the fibre's ability to produce force, the maximum speed of shortening does not depend on the number of myosin bridges that are able to interact with the thin filaments. The results illustrated in Fig. $6 B$ provide evidence that $V_{\max }$ is also independent of the degree of activation of the contractile system. $\mathrm{V}_{\max }$ during a twitch is thus virtually the same as during tetanus, even after depressing the twitch force to less than $10 \%$ of the control isometric 
tetanus by dantrolene, an agent known to depress the release of activator calcium from the sarcoplasmic reticulum.

\section{The slack test method. Braking force of cross-bridges at negative strain}

A standard approach in the past to gain information on the maximum speed of shortening of muscle has been to extrapolate from velocity measurements performed at finite loads. However, the steepness of the force-velocity curve in the low-force range makes this approach uncertain and typically leads to underestimation of the highest shortening speed that the muscle is capable of. A convenient method of measuring the speed of shortening at zero load was originally described for use on intact single muscle fibres (Edman, 1979) but the approach has been found useful in other situations as well, both in intact and skinned preparations of skeletal, cardiac and smooth muscles (see further Reggiani, 2007).



Fig. 7. Superimposed records from a single muscle fibre showing three releases of different amplitudes during plateau of tetanus. Upper traces: tension. Lower traces: release steps calibrated in $\mu \mathrm{m}$ sarcomere length. From Edman (1979).

The method, here referred to as the slack test method, has certain features that are of interest to point out. The preparation is quickly released during activity (Fig. 7) so as to rapidly slacken the preparation to cause an abrupt fall in tension to zero, thereafter allowing the preparation to shorten actively to take up the slack. Three or more amplitudes of release $(\Delta L)$ 
are used, care being taken to ensure that the smallest release is more than sufficient to slacken the series elasticity. The time $(\Delta t)$ measured from the onset of release to the

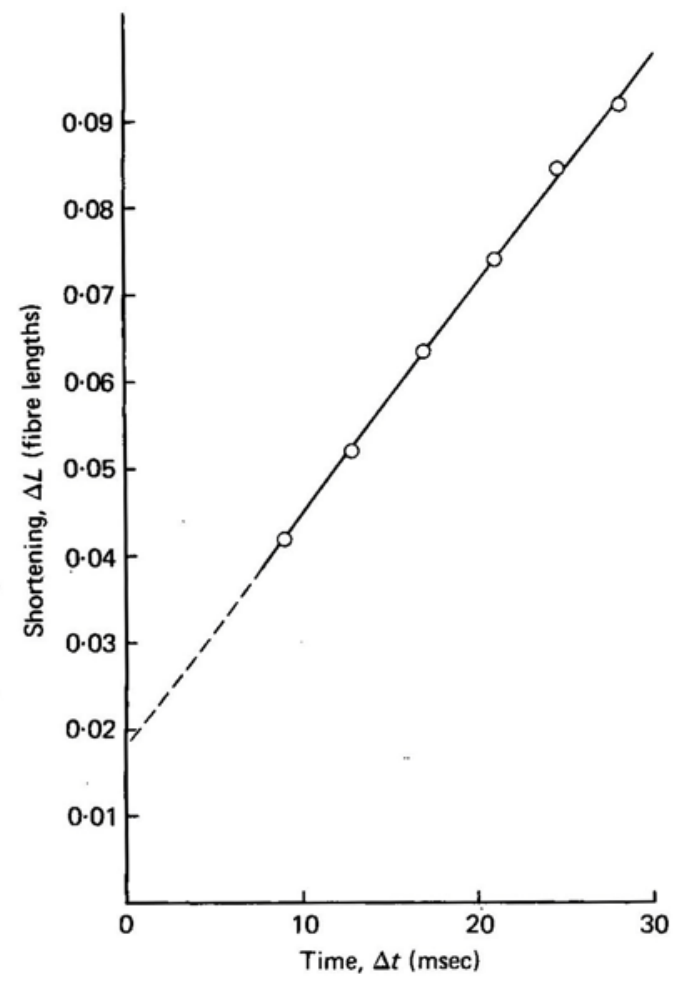

Fig. 8. Relationship between amplitude of shortening $(\Delta L)$ and time from onset of release to beginning of force redevelopment $(\Delta t)$. Releases performed from $2.25 \mu \mathrm{m}$ sarcomere length during plateau of tetanus as illustrated in Fig. 7. Each data point is the mean of five release recordings. Straight line: least-squares regression of $\Delta t$ upon $\Delta L$. Intersection of line with ordinate: total series compliance of preparation during isometric tetanus. From Edman (1979).

onset of force redevelopment is plotted against the amplitude of the release movement $(\Delta L)$ as shown in Fig. 8. The slope of the straight line so derived (regression analysis) provides a measure of the speed of shortening at zero load. By this approach it is possible to exclude any contribution from the recoil of series elastic components that occurs when the muscle is released and the tension drops to zero. The magnitude of the total series compliance is provided in the slack test measurement by extrapolating the line relating $\Delta L$ and $\Delta t$ to zero time (Fig. 8).

The slack test method performed at an extended fibre length provides compelling evidence that the myosin cross-bridges are able to exert a braking force when bridges are brought into negative strain. This is demonstrated in Fig. 9, which shows, superimposed, three release 
steps of different amplitudes (lower panel) ending up at a given sarcomere length $(2.95 \mu \mathrm{m})$ in each case. The resulting changes in force are shown in Fig. $9 \mathrm{~A}$ when the release steps are carried out at rest. The results show that each release step results in a rapid fall in resting tension and that the tension is maintained quite constant at the new level. By contrast, as shown in Fig. 9 B, when the same releases are carried out during activity the tension drops to zero, i.e. the response is similar to that recorded at a short sarcomere length where no resting tension exists (cf. Fig. 7).

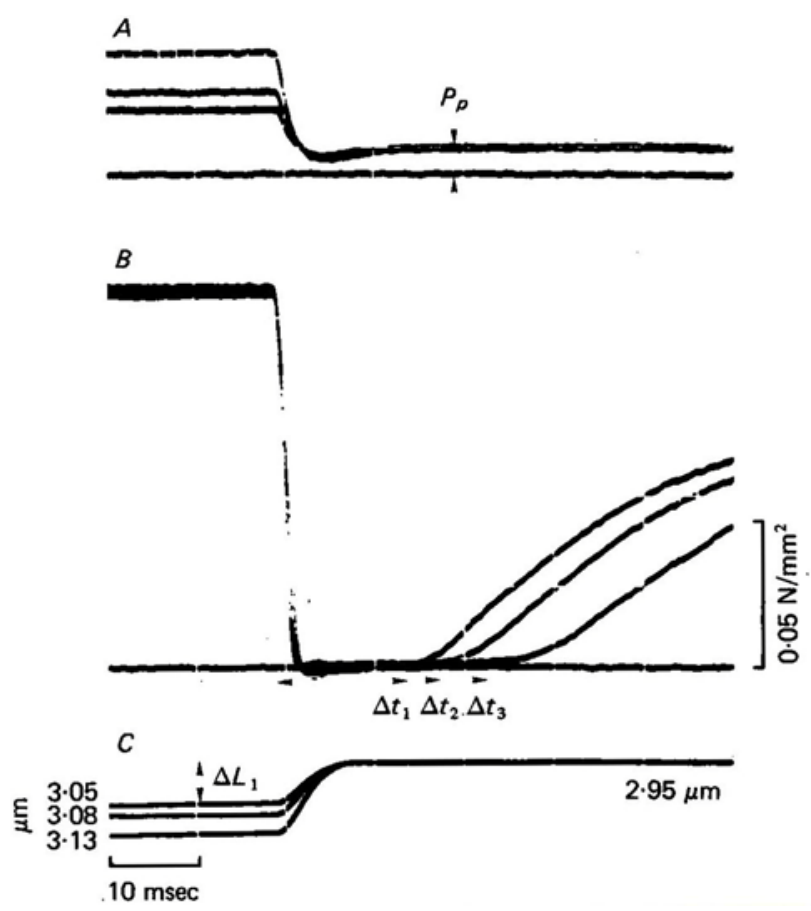

Fig.9. Superimposed quick release records illustrating changes in force when the release steps are carried out at rest $(A)$ and during the plateau of tetanus $(B)$ in a prestretched fibre. The release steps $(C)$ indicate the sarcomere length before and after release. Note that the fibre holds a finite resting tension $(A)$ after the release steps whereas tension is reduced to zero when the fibre is quickly released during tetanus $(B)$. See text for further explanation. From Edman (1979).

The finding that tension is reduced to zero in the active fibre in spite of the fact that the parallel elastic elements are still under strain after the release steps (Fig. 9 A), provides evidence that the passive force exerted by the parallel elastic elements is absorbed by the myofibrils: A fraction of active cross-bridges may thus be presumed to go into negative strain 
after the release in this way neutralizing the compressive force produced by the parallel elastic elements.

\section{Force enhancement by stretch}

When striated muscle is stretched during activity there is an initial rapid rise of force that reaches a breakpoint after which the force remains essentially constant or, if the muscle is stretched beyond its optimal length, increases slowly during the remainder of the stretch period. This phenomenon is referred to as 'force enhancement during stretch' and has been investigated in several previous studies, both in whole muscle (Fenn, 1924; Abbott \& Aubert, 1952: Hill \& Howarth, 1959; Cavagna \& Citterio, 1974) and in single fibre preparations (Sugi, 1972, Edman et al. 1978, Julian \& Morgan, 1979; Edman, 1999). The amplitude of force attained during stretch increases with the velocity of stretch, and a plot of the enhanced force against the stretch velocity exhibits the well-known 'negative' branch of the forcevelocity relationship in skeletal muscle (Katz, 1939; Aubert, 1956, Edman, 1988). The force enhancement during stretch gradually disappears after the end of stretch and reaches a stable level within 4-5 s in a single muscle fibre at low temperature (Fig. $10 \mathrm{~A}$ and $\mathrm{B}$ ). It is noteworthy, however, that if the stretch has been performed above optimal length, the final tension remains clearly higher than the control isometric tetanus at the stretched length. This remaining component of force increase is independent of the stretch velocity and is referred to as ‘residual force enhancement after stretch’ (Edman et al. 1978). 
$A$

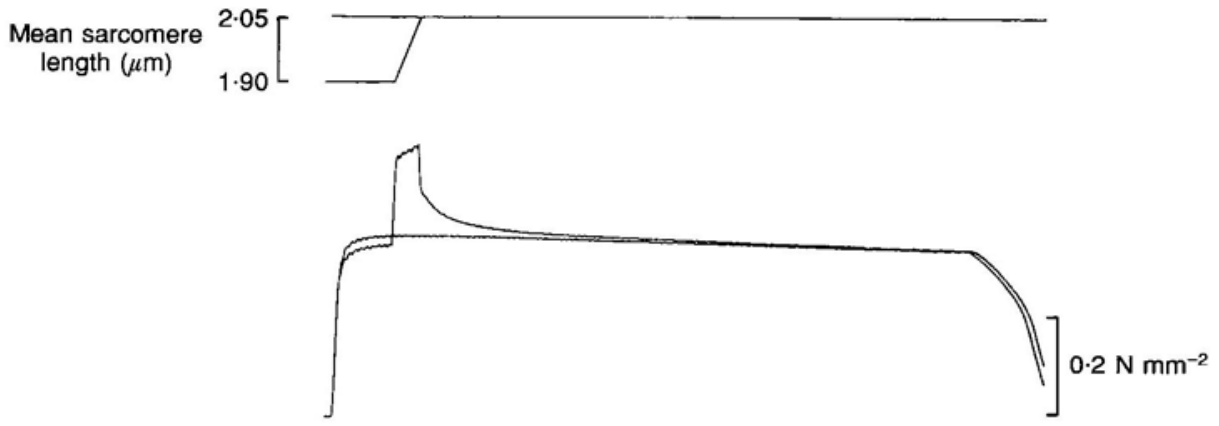

$B$

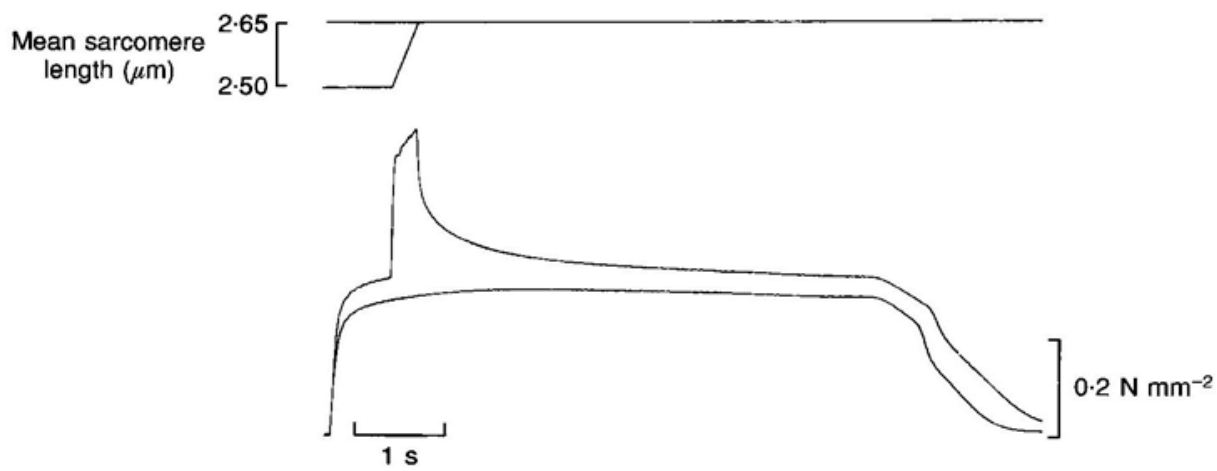

Fig. 10. Force enhancement by stretch during tetanus of a single muscle fibre at two different sarcomere lengths.

A, stretch during activity from 1.90 to $2.05 \mu \mathrm{m}$ sarcomere length compared with ordinary isometric tetanus at $2.05 \mu \mathrm{m}$.

$B$, stretch from 2.50 to $2.65 \mu \mathrm{m}$ sarcomere length compared with isometric tetanus at $2.65 \mu \mathrm{m}$.

Note that the same force level is finally reached in both records in $A$, whereas force after stretch remains above the isometric control level throughout the activity period in B. From Edman \& Tsuchiya (1996).

\section{Force enhancement during stretch, its relation to sarcomere length and myofilament lattice width}

The force enhancement during stretch is likely to mark the point at which the cross-bridges

can no longer withstand the external pull and therefore start to slide along the thin filament.

In a study of length controlled segments of intact muscle fibres it was demonstrated (Edman, 1999) that the amplitude of force enhancement during stretch varies with sarcomere length

but in a way that is markedly different from that of the plain isometric force. 
. Fig. 11 illustrates oscilloscope records from a length-controlled segment of an intact muscle fibre that is subjected to stretch during the plateau of an isometric tetanus. The stretching can be seen to cause a rapid rise of tension to a breakpoint after which the tension remains constant during the remainder of the stretch period. The force enhancement considered here is the rise of force above the isometric level ending at the breakpoint of the force myogram (marked $b$ in Fig. 11).

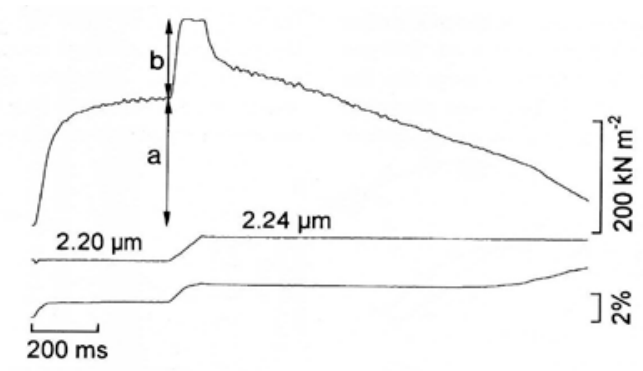

Fig. 11. Force and displacement records from a length-controlled segment of an intact muscle fibre subjected to stretch on the plateau of a fused tetanus. Upper trace, force; middle trace, segment length calibrated in $\mu \mathrm{m}$ per sarcomere; lower trace, change of puller position as required to adjust the segment length. Note the rapid rise of force after the onset of stretch ending at a breakpoint after which the force remains quite constant during the rest of the stretch ramp. Stretch amplitude at the breakpoint of the force myogram is approximately $8 \mathrm{~nm}$ per half sarcomere. a, isometric force; $b$, measured force enhancement. From Edman (1999).

As demonstrated in Fig. 12 A (filled symbols), force enhancement during stretch increases with sarcomere length above $1.8 \mu \mathrm{m}$ reaching a maximum at approximately $2.4 \mu \mathrm{m}$, at which point the enhanced force can be seen to be equally large as the isometric force. With further increase in sarcomere length the force enhancement during stretch becomes gradually smaller. 

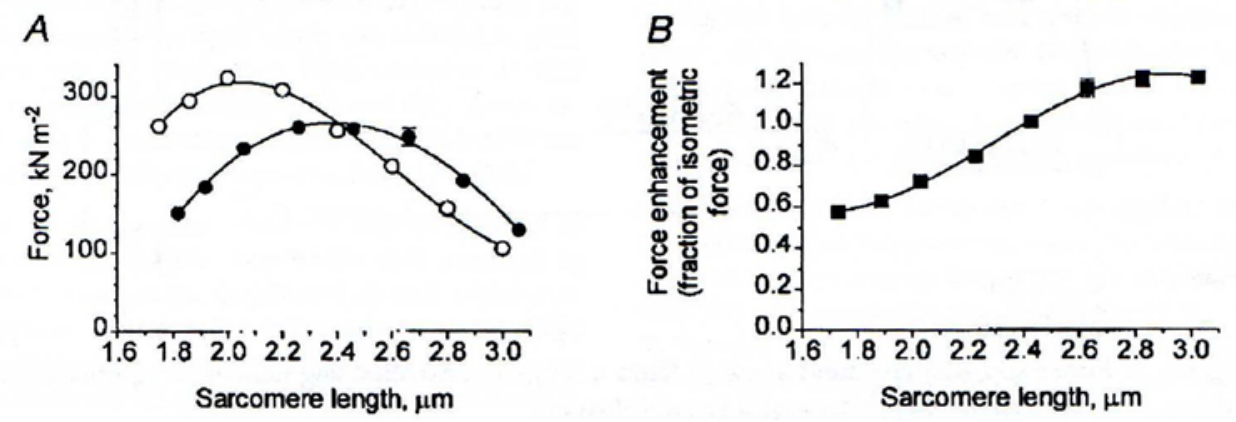

Fig. 12. Isometric tetanic force and force enhancement during stretch in relation to sarcomere length. $A$, measurements of tetanic force (Open circles) and force enhancement during stretch (Filled circles) recorded from a given length-controlled segment of a single muscle fibre. Mean values of five or six repeated recordings at each sarcomere length. Error bars (if exceeding symbols) indicate S.E.M. From Edman (1999). $B$, force enhancement during stretch normalized to the tetanic force recorded at respective sarcomere length. From Edman, 1999.

Fig. 12B shows the data from Fig. 12A replotted as a fraction of the control isometric force recorded at the various sarcomere lengths in this way normalizing the force enhancement to the amount of filament overlap at each sarcomere length. Pooled data from seven experiments are shown in Fig 13. The force enhancement during stretch can be seen to increase steadily, relative to the isometric force, as the sarcomere length is changed from approximately 1.8 to $2.8 \mu \mathrm{m}$ sarcomere length. This finding strongly suggests that the width of the myofilament lattice is an important determinant of the force enhancement during stretch given the fact that an intact muscle fibre behaves as a closed volume (see further below). The relative fibre width at the different sarcomere lengths is indicated above the abscissa in Fig. 13 and has been calculated on the assumption that the myofilament lattice maintains a constant volume within the range of sarcomere lengths considered (Elliott et al., 1963; Huxley, 1969; Millman 1998). 


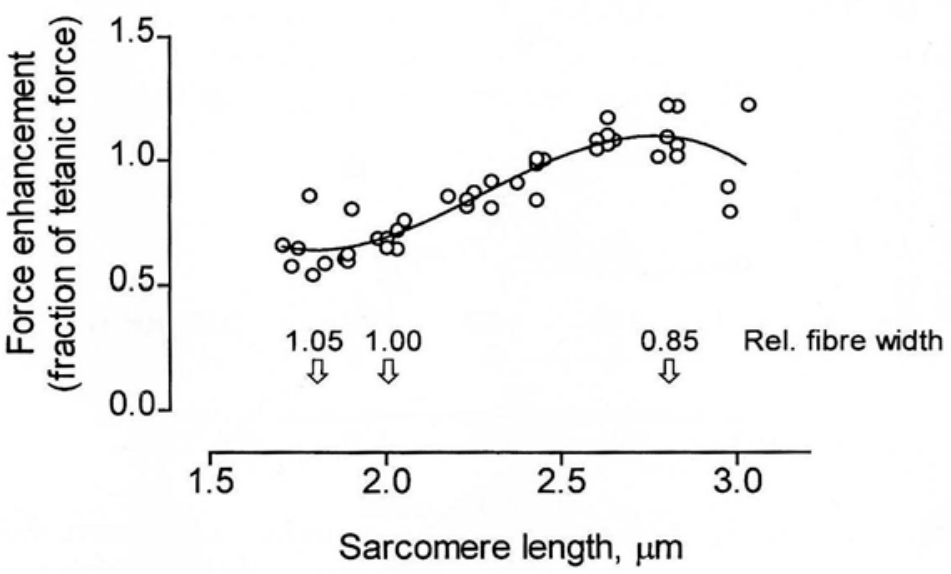

Fig. 13. Force enhancement during stretch (normalized to tetanic force) related to sarcomere length and fibre width.

Pooled data from seven experiments similar to that illustrated in Fig. 12B. The relative fibre width at different sarcomere length is indicated above the abscissa. From Edman (1999).

The data in Fig. 13 show that a decrease in relative fibre width below the value (1.05) existing at $1.8 \mu \mathrm{m}$ sarcomere length is associated with a steady increase of the normalized force enhancement during stretch. Maximum force enhancement is attained near $2.8 \mu \mathrm{m}$ sarcomere length at which point the relative fibre width is estimated to be merely 0.85 of the value at $2.0 \mu \mathrm{m}$ sarcomere length. The tighter packing of the myofilament lattice at the greater sarcomere length apparently makes the myosin cross-bridges more capable to withstand stretch; the resistive force can be seen to increase by approximately $65 \%$ by increasing the sarcomere length from approximately 1.8 to $2.8 \mu \mathrm{m}$ (Fig. 13). This width dependence of the force enhancement during stretch was confirmed in the same study by varying the fibre width over a similar range by altering the tonicity of the extracellular medium (for details, see Edman, 1999).

The above results suggest that the strength by which the myosin bridges bind to the thin filaments during activity is greatly dependent on the width of the myofilament lattice. It is of interest to note in this connection that the myosin head (approximately $17 \mathrm{~nm}$ long and $6 \mathrm{~nm}$ wide; Elliott \& Offer, 1978; Rayment et al, 1993) is large enough to fill the entire gap 
between the thick and thin filaments near slack fibre length, the side-to-side distance between the two filaments being $17.2 \mathrm{~nm}$ at $1.8 \mu \mathrm{m}$ sarcomere length. (The estimation of the interfilament spacing is based on published data on the 1.0 lattice spacing of the X-ray diffraction pattern and on electron microscopical measurements of filament diameters; for details see Edman, 1999). The free space available for the myosin head will become drastically reduced when the sarcomere length is increased above slack length. At $2.8 \mu \mathrm{m}$ sarcomere length the side-to-side distance between the thick and thin filaments can be estimated to be merely $11.9 \mathrm{~nm}$. The tighter packing of the myofilaments at greater sarcomere lengths can be presumed to increase the contact area between the actin and myosin molecules. This may increase the opportunities for bond formation between the two structures and lead to firmer attachment of the bridge. Another corollary of the limited space between the filaments is that the shaft of the myosin bridge (the S2 region) will stay virtually parallel with the actin filament over the entire working range of sarcomere lengths. This is fully in line with the earlier conclusion that the force producing capability of striated muscle is a function of the length of filament overlap per se with no relation to the myofilament lattice width (Edman \& Andersson, 1968).

\section{Residual force enhancement after stretch}

The longlasting component of force enhancement by stretch (Fig. 10B) that remains at the end of a long (5-10 s) tetanus was originally observed on isolated whole muscles of the frog (Fenn, 1924; Abbott \& Aubert, 1952; Hill \& Howarth, 1959) and has later been subjected to detailed studies on isolated single muscle fibres (Edman et al., 1978, 1982; Sugi, 1972; Edman \& Tsuchiya, 1996, Sugi \& Tsuchiya, 1988; Julian \& Morgan, 1979; Rassier \& Herzog, 2004a, 2004b, Herzog \& Rassier, 2006) and, recently, on isolated cardiac and skeletal muscle myofibrils (Telley et al., 2006; Joumaa et al., 2008; Rassier et al., 2003). The 
continued interest in this phenomenon is based on the fact that the longlasting force enhancement by stretch does not seem to be readily explainable on the basis of the slidingfilament hypothesis. The muscle fibre is, however, a complex mechanical structure, to say the least, and there is so far no convincing evidence that the residual force enhancement after stretch represents a phenomenon that is not ultimately based on the sliding-filament model.

In early studies on intact single muscle fibres (Edman, Elzinga Noble, 1978, 1982) a number of salient features of the phenomenon 'force enhancement after stretch' were described. The magnitude of force enhancement was found to be independent of the stretch velocity, to increase with the amplitude of stretch and to occur on the descending limb of the length-tension relationship only. It was found that the absolute magnitude of the residual force enhancement increased with sarcomere length to a maximum at $\sim 2.9 \mu \mathrm{m}$ and then declined. Particular attention was paid to the question of whether the residual force enhancement after stretch might exceed the control isometric force at optimal sarcomere length. Our results showed that stretches causing a marked force enhancement above optimal length, did not clearly increase the force when performed on the plateau of the isometric length-tension relationship (Edman et al., 1982).

The notion that force enhancement after stretch is based on non-uniform sarcomere behaviour in response to stretch is supported by electron microscopical studies in which muscle fibres have been rapidly fixed after a stretch ramp. Brown \& Hill (1991) were first to demonstrate marked irregularities of myofilament overlap within myofibrillar sarcomeres along the muscle fibre after a stretch ramp. That is, in spite of an overall elongation of the sarcomere pattern in response to the stretch the degree of filament overlap may differ between individual sarcomere halves. Thus, the amount of filament overlap in one half of the sarcomere may actually become larger during and after stretch at the expense of the filament overlap in the opposite half of the sarcomere which becomes correspondingly smaller. The 
uneven distribution of filament overlap after stretch has been confirmed in other similar studies (Edman \& Lou, unpublished data based on a rapid-freezing technique; Edman \& Lou, 1992; Talbot \& Morgan, 1996).

An essential component of the mechanism of residual force enhancement after stretch is the development of strained elastic elements during the stretch process. This particular problem was addressed by Edman \& Tsuchiya (1996) in a study of frog muscle fibres with simultaneous measurements of length and force from the fibre as a whole and from marked segments of the same fibre. The existence of strained elastic element along the fibre during tetanic contraction manifested itself in a damped initial transient when the fibre was released to shorten against a very small load at different times after a stretch ramp. The initial transient increased linearly with the amplitude of the preceding stretch and with the force enhancement existing at the moment of release (for details, see Fig. 4 in Edman \& Tsuchiya, 1996). The evidence presented by Edman \& Tsuchiya suggests that strain of passive elastic elements along the fibre does not in itself explain the extra force recorded during and after stretch. The extended elastic elements are likely to be dispersed within the fibre volume and merely serve to support the weakened parts of the myofibrils (parts with reduced filament overlap) to enable them to match the stronger parts of the fibre during and after stretch. According to this view the measured force enhancement after stretch is thus actively produced by those sarcomeres, or sarcomere halves, that have acquired a more favourable degree of filament overlap during stretch than other regions in series.

Consistent with this view no residual force enhancement after stretch would be expected when the fibre is stretched over the "plateau" of the length-tension relation. The strongest regions after stretch would already be at optimal filament overlap and so would not achieve a greater force after stretch than that produced in the isometric control (however, see below). Results reported by Edman et al. (1982), based on a large number of experiments, support this 
prediction by showing that the residual force after stretch was not clearly above the plateau of the length-tension curve. This problem has recently been revisited by Lee \& Herzog (2008) who conclude that a stretch ramp carried out within the plateau of the length-tension relation may result in a small (4.3 \%) but distinct residual force enhancement.

The effect described by Lee \& Herzog (2008) by no means disqualifies the idea that force enhancement after stretch is based on non-uniform sarcomere behaviour. Firstly the lengthtension relationship in frog muscle fibres does not have a perfectly flat maximum between 2.0 and $2.2 \mu \mathrm{m}$ sarcomere length as early studies seemed to suggest (Edman, 1966; Gordon et al., 1966). In reality there is a steady increase of the tetanic force of approximately $7 \%$, as the overall sarcomere length is changed from 2.2 to $1.95 \mu \mathrm{m}$ as demonstrated in an extensive study on short, length-controlled segments of isolated frog muscle fibres (Edman \& Reggiani, 1987). The absence of a real plateau of the length-tension relation can be explained by the fact that the thick and thin filaments are not constant in length and, furthermore, that there is normally some staggering (longitudinal misalignment) of the filaments within the A and I bands in a given fibre cross-section (See further Edman \& Reggiani, 1987). To this may be added that the force producing capacity may differ by several (up to 10) \% along the length of the muscle fibre (Edman et al., 1985). Taken together these findings imply that one or more regions of the fibre are actually capable of producing a somewhat greater force than that recorded from the fibre as a whole within the "plateau" of the length-tension relation (see Edman \& Reggiani, 1987). During stretch, on the other hand, it is the strongest regions of the muscle fibre that alone determine the force recorded from the fibre. The weaker parts in series will match the weaker segments by recruiting supportive elastic components as described in some detail by Edman \& Tsuchiya (1996). 


\section{Force reduction after loaded shortening}

It has been known for a long time (Abbott \& Aubert, 1952) that striated muscle that is released to shorten against a high load during tetanus produces less force after the end of shortening than during a fixed-end tetanus at the corresponding length (Marechal \& Plaghki, 1979). Fig. 14 illustrates the force depression after loaded shortening in a single muscle fibre (A) and, for comparison, the lack of force depression when the same amount of shortening occurs without load $(B)$. Non-uniform sarcomere behaviour was early considered as one possible cause
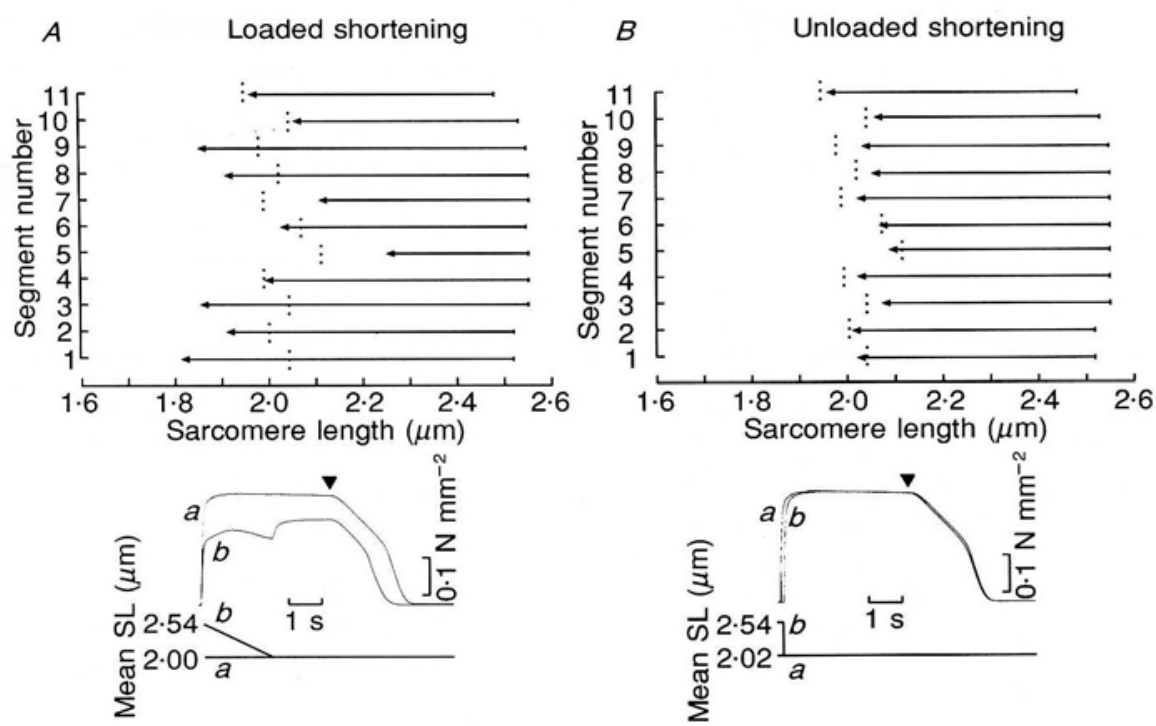

Fig. 14. Differential length changes along a single muscle fibre during tetanus with loaded $(A)$ and unloaded $(B)$ shortening, respectively. The change in sarcomere length from rest to the end of the tetanus plateau (triangle above myograms) is indicated by horizontal arrows for 11 consecutive segments. Dotted vertical lines indicate the sarcomere length in the same segments during fixed-end tetanus at the short fibre length.

The superimposed records below the diagrams show force and puller position during control tetanus at short length (myograms $a$ ) and during tetanus with shortening (myograms $b$ ). Note that loaded shortening leads to a marked force depression and a wide distribution of sarcomere length. By contrast, unloaded shortening does not result in force depression and the sarcomere pattern after unloaded shortening is not markedly different from that of a fixed-end tetanus. From Edman et al. (1993).

of the force deficit, an explanation that gained direct experimental support by Julian \& Morgan (1979) in studies of single muscle fibres. However, there has been a continuous search for alternative explanations and several imaginative mechanisms have been proposed (e.g. Maréchal \& Plaghki, 1979; Sugi \& Tsuchiya, 1988; Granzier \& Pollack, 1989; Schachar 
et al., 2004). The phenomenon of force depression by loaded shortening was revisited by Edman, Caputo \& Lou (1993) in frog single muscle fibres using a combination of supplementary techniques to elucidate the possible role of non-uniform sarcomere behaviour. The following observations were made:

i. the force deficit after loaded shortening is quantitatively related to the variation in sarcomere shortening along the length of the fibre. The interrelationship between force depression and sarcomere non-uniformity after loaded shortening is exemplified in Fig. 14 and is presented statistically in Fig.15.

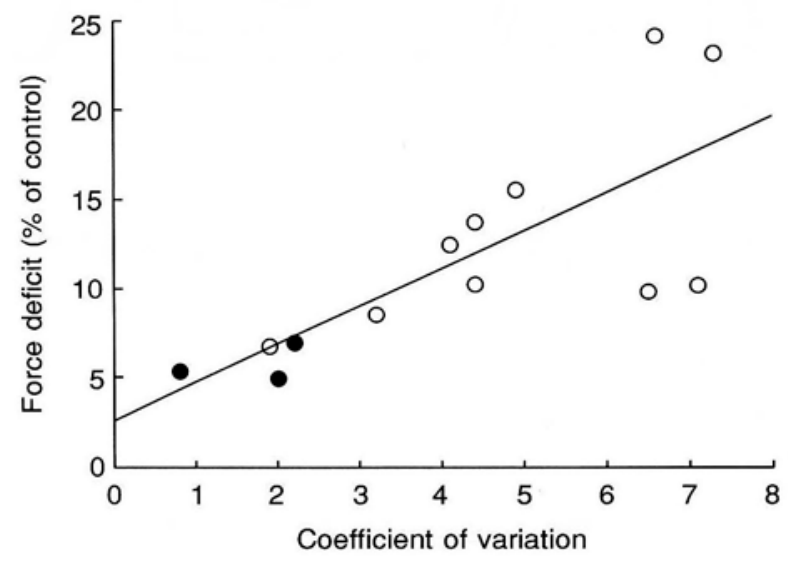

Fig. 15. Relation between force deficit after loaded shortening $(\Delta F)$ and coefficient of variation $\left(K_{\mathrm{v}}\right)$ of sarcomere length along the muscle fibre. $K_{\mathrm{v}}$ is the standard deviation expressed as percentage of the mean. Open circles, shortening above slack length; filled circles, shortening below slack length. Regression line $(\mathrm{P}<0.005)$ : $\Delta F=2.10 K_{\mathrm{v}}+2.55$. From Edman et al. (1993).

ii. The force depression after loaded shortening disappears after imposing a brief (10

s) period of relaxation (Fig. 16). Furthermore, as illustrated in Fig. 16, the recovery of force after relaxation was associated with the reappearance of a quite uniform sarcomere pattern along the fibre. 

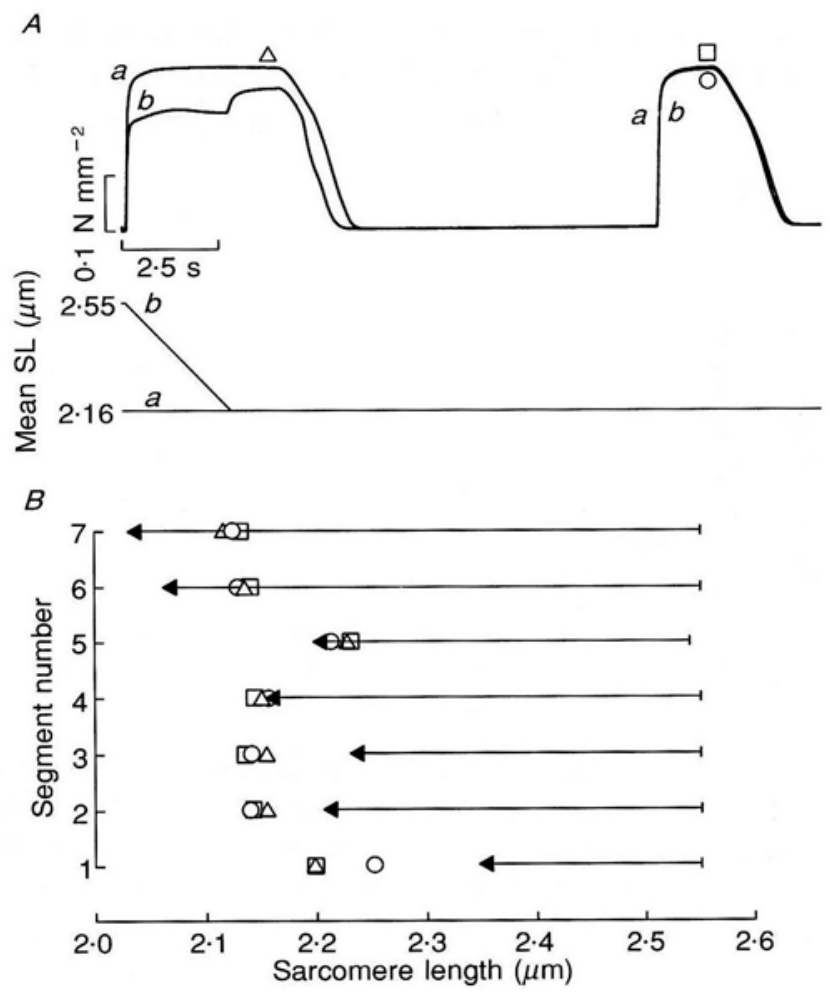

Fig. 16. Disappearance of force depression after loaded shortening by interposing a brief period of relaxation. The superimposed myograms in $A$ show the following stimulation sequence which was repeated every 4 min: $4 \mathrm{~s}$ tetanic stimulation followed by a $10 \mathrm{~s}$ pause and a new , $1.5 \mathrm{~s}$ period of tetanic stimulation. In myogram $b$ the fibre was allowed to shorten against a high load from a mean sarcomere length of $2.55 \mu \mathrm{m}$ to $2.16 \mu \mathrm{m}$ during the $4 \mathrm{~s}$ tetanus. In myogram $a$ (control) the fibre was held at the short length throughout. Note that the force deficit produced by loaded shortening during the first tetanus is no longer seen when tetanization is resumed after relaxation. The horizontal arrows in $B$ show the change in sarcomere length of successive segments along the fibre during the $4 \mathrm{~s}$ tetanus with loaded shortening. Open triangles, open squares and open circles indicate the sarcomere length in the same segments at the times shown at respective force myogram in $A$. Note that the force deficit after loaded shortening is associated with a grossly irregular sarcomere pattern along the fibre and that the length pattern returns to normal, together with restoration of the tetanic force, as the fibre is restimulated after a brief period of relaxation. From Edman et al. (1993).

iii. No force deficit occurs after loaded shortening when recording is made from the short length-controlled segment of a single muscle fibre. This finding holds true either the loaded shortening occurs above or below slack length (Fig. 17). 

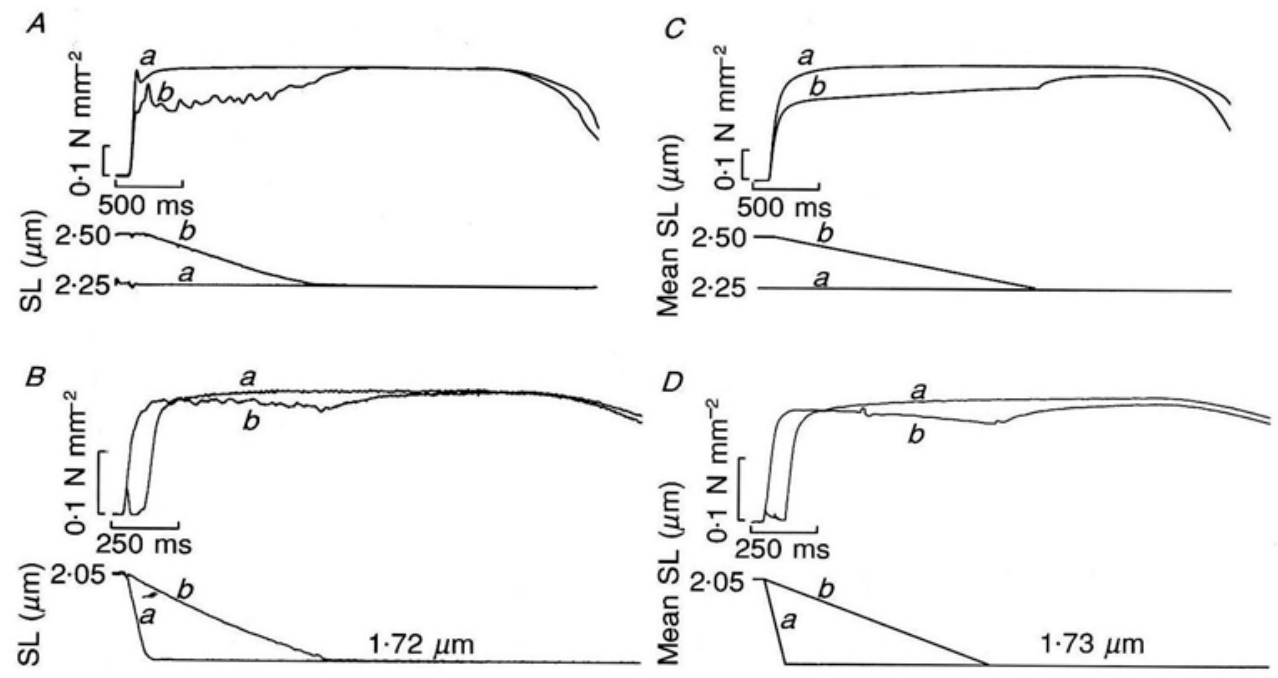

Fig. 17. Lack of force deficit after loaded shortening of length-controlled segment of single muscle fibres above $(A)$ and below $(B)$ slack length. $C$ and $D$ show, for comparison, the effects of loaded shortening when recording is made from the fibre as a whole. The same fibre in $A$ and $C$ and in $B$ and $D$, respectively. Myograms $a$, control tetani; myograms $b$, tetani with loaded shortening. Control tetani below slack length contain an initial phase of unloaded shortening. From Edman et al., (1993).

iv. A computer model of a typical fibre with non-uniform sarcomere behaviour simulates the force deficit after loaded shortening exceedingly well. For details, see Edman, Caputo and Lou, 1993.

Taken together the above results would seem to leave little doubt that non-uniform sarcomere behaviour is the main cause of the force deficit after loaded shortening in striated muscle. It is essential to point out that the force depression so produced is distinct from the transitory deactivation by active shortening to be described next.

\section{Deactivation by active shortening}

When striated muscle is allowed to shorten during activity it loses temporarily some of its capacity to produce force. This depressant effect of shortening has been demonstrated in both isolated muscle preparations (Jewell \& Wilkie, 1960; Edman \& Kiessling, 1971; Briden \& Alpert, 1972) and muscles in situ in the body (Joyce et al., 1969). This phenomenon, which 
is unrelated to fatigue, has previously been studied in considerable detail on both intact and skinned muscle fibres (Edman, 1975, 1980, 1981; Ekelund \& Edman 1982).

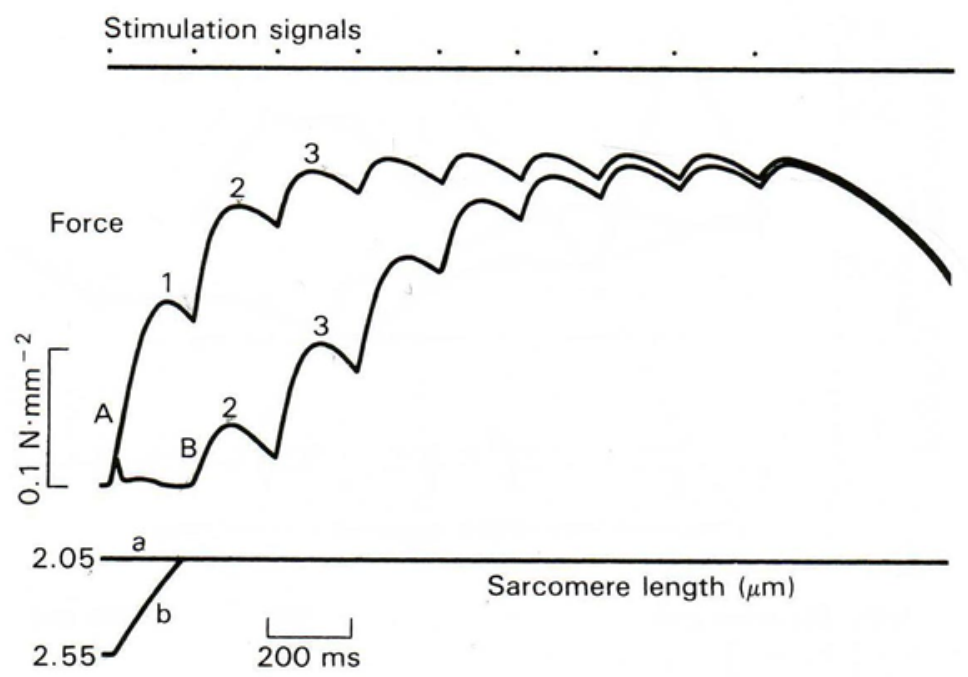

Fig. 18. Transitory depressant effect of shortening during partially fused tetanus of a frog muscle fibre. The superimposed myograms $A$ and $B$ show force development at $2.05 \mu \mathrm{m}$ sarcomere length. In myogram $B$ contraction is initiated at $2.55 \mu \mathrm{m}$ sarcomere length and the fibre is allowed to shorten to $2.05 \mu \mathrm{m}$ during the first twitch cycle as indicated by the length record underneath. The first few twitch cycles in the myograms are numbered for identification in the text.

The depressant effect of active shortening is demonstrated in Fig. 18 which illustrates two superimposed records of a partially fused tetanus in a frog single muscle fibre. Record A (control) shows the development of force at $2.05 \mu \mathrm{m}$ sarcomere length when the fibre ends are fixed throughout the entire stimulation period. In record B the same stimulation sequence is repeated, but here the fibre is initially set at a longer sarcomere length, $2.55 \mu \mathrm{m}$, and the fibre allowed to shorten to $2.05 \mu \mathrm{m}$ during the first twitch period. The fibre is arrested at this length to develop force during the remainder of the stimulation period, i.e. at the same length as in the control run. The records in Fig. 18 clearly demonstrate that force development is depressed after the shortening phase. Twitch No. 2 in myogram B can thus be seen to be considerably lower than the first twitch in the control run. This is significant since tension starts from zero level and occurs at the same sarcomere length in both cases. The lower force recorded in twitch period No. 2 in myogram B thus represents a true depression of the fibre's 
capacity to produce force, due to the preceding shortening. It is clear, however, that the depressant effect of shortening disappears with time during continued stimulation. Full strength can be seen to be regained 1-2 s after the end of the shortening phase.


Fig. 19. Depressant effect of active shortening tested at different states of activity of a single muscle fibre. Control $(a)$ and test $(b)$ movements performed during the last cycle of partially fused tetanus $(A-C)$ and prior to the last stimulus of fused tetanus $(D-F)$. A, $D$ : fibre immersed in ordinary Ringer solution. $B, E$ : 45-65 min after addition of $0.5 \mathrm{mM}$ caffeine to the bathing fluid. $C, F$ : 50-65 min after change to ordinary Ringer solution.

Lower traces in each panel indicate sarcomere length before and after the shortening. Temperature: $2.5^{\circ} \mathrm{C}$. From Edman (1980). 
There is evidence to suggest that shortening temporarily reduces the degree of activation of the contractile system. In line with this view the depressant effect of shortening is diminished if the state of activity in the fibre is raised. The top left panel $(A)$ of Fig. 19, illustrates the depressant effect in a partially fused tetanus. Myogram a (control) of Fig. 19 shows the redevelopment of force after a small shortening which is just sufficient to produce a drop in tension and an immediate redevelopment of force. In myogram $b$ the amplitude of shortening is larger, and the release is timed so as to make the redevelopment of force start at the same time as in the control. The additional amount of shortening produced in myogram $b$ can be seen to depress the amplitude of the redeveloped force by approximately $25 \%$. Panel $D$ shows, for comparison, the corresponding effect of shortening during a fused tetanus in the same fibre, i.e. under conditions when the contractile system can be presumed to be fully activated. The shortening is here produced during the plateau of the tetanus, before the last stimulus. The resulting depression of force (approximately $7 \%$ ) can now be seen to be considerably smaller than during the partially fused tetanus.

The middle panels ( $B$ and $E$ ) of Fig. 19 shows that the depressant effect of shortening is much reduced in the presence of caffeine in a concentration that is known to increase the release of calcium from the sarcoplasmic reticulum in amphibian muscle (Blinks et al. 1978). The depressant effect of shortening can be seen to be greatly reduced in the presence of caffeine, both in absolute and relative terms, during the partially fused tetanus, and the movement effect is almost abolished during the fused tetanus. Finally, the depressant effect of shortening is fully restored after removal of caffeine from the bathing fluid (Panels $C$ and $F$ ).

The above results support the view that active shortening temporarily reduces the state of activation of the contractile system, most likely by reducing the affinity of calcium at its binding-sites on troponin (see further below). In line with this view, the movement effect is large when the contractile system is submaximally activated as is the case during a twitch and 
a partially fused tetanus. This interpretation of the movement effect is also strongly supported by the finding that there is virtually no force depression by shortening when the contractile system is maximally, or supramaximally, activated which is the case during a fused tetanus in the presence of caffeine. Under these circumstances a decrease in calcium affinity at the binding sites would be compensated by a higher free calcium concentration in the myofibrillar space.
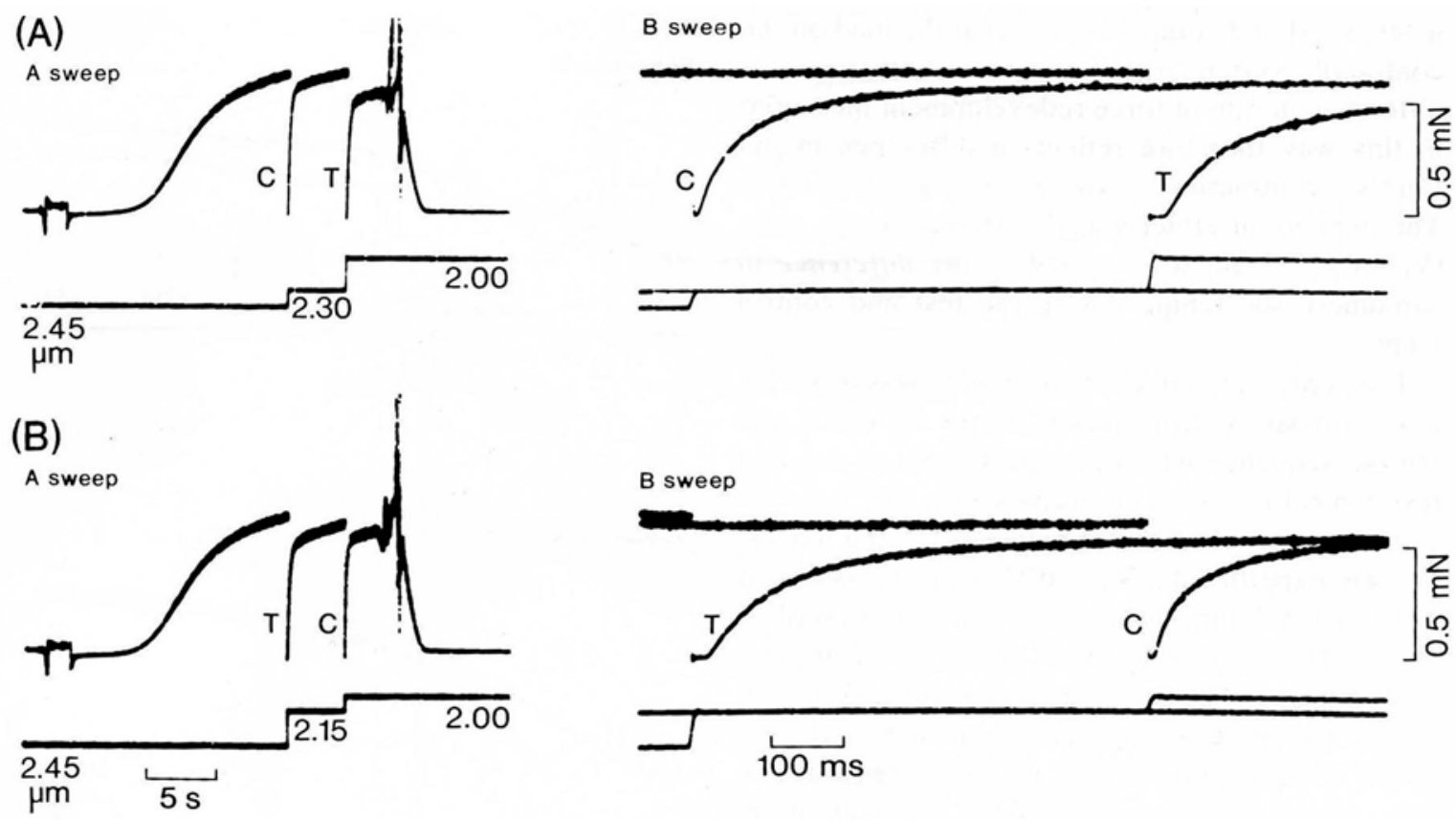

Fig. 20. Depressant effect of active shortening during calcium induced contraction of chemically skinned fibre of the mouse. Small (control) and large (test) release steps, designated C and T, respectively, carried out in sequence during the same contraction. The order of $C$ and $T$ reversed in $A$ and $B$. The right-hand traces show the same release recordings on a faster time base. Note that the rate of force redevelopment is smaller after the large (test) release. From Ekelund \& Edman (1982).

Experiments on skinned muscle fibres from frog and mouse provide further support for the view that the movement effect is based on a change in the myofilament system that leads to a temporary decrease in mechanical activity. The approach used is illustrated in Fig. 20 which shows the redevelopment of force after a small (control) and large (test) release step (C and T, respectively) during a calcium induced contraction of a skinned muscle fibre of the mouse. The records readily show that the rate of force redevelopment is lower 


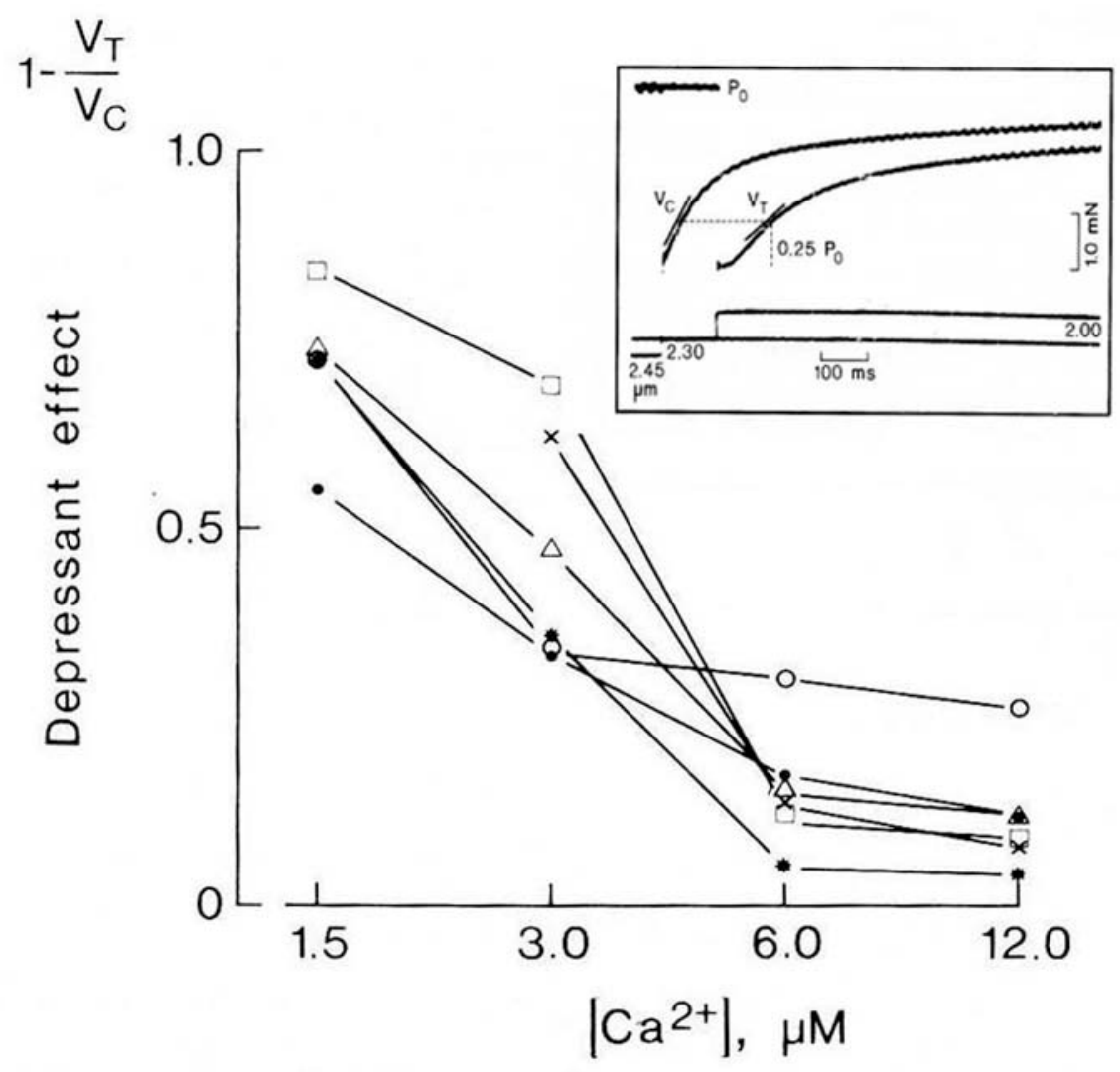

Fig. 21. Depressant effect of active shortening of skinned muscle fibres of the mouse related to the free calcium concentration. The depressant effect was measured as the decrease in the rate of force redevelopment at $25 \%$ of maximum isometric force as illustrated in the inset. Each data set denoted by the same symbol is from a given fibre preparation. From Ekelund \& Edman (1982).

after the larger shortening step. However, the same final tension was finally attained after both amplitudes of release. For quantification of the depressant effect of shortening the rate of force redevelopment, at $25 \%$ of maximum force, was used as an index as shown in Fig. 21 (inset). The fact that the measurements refer to the same tension level in test and control implies that the load on the contractile system was the same in both cases. As illustrated in Fig. 21 (main diagram), an increase in free calcium concentration from 1.5 to 6-12 $\mu \mathrm{M}$ greatly reduced the depressant effect of shortening.

The evidence suggests that active sliding of the myofilaments leads to a decrease in activation of the contractile system. Activity is needed for the depressant effect to occur. That is, the two filaments have to interact during sliding for the movement effect to occur (Edman, 1980). These findings support the view (Edman, 1975; Ekelund \& Edman, 1982) that the 
myosin bridges interfere with the regulatory proteins as the bridges interact with the thin filaments during muscle shortening. The movement effect may actually be a manifestation of a cooperative activity between the proteins of the calcium-regulated thin filament leading to a transitory decrease in calcium affinity and, therefore, to a transient deactivation of the contractile machinery. In line with this idea, there is a transitory increase in the myoplasmic calcium ion concentration during the shortening process, suggesting that calcium previously bound to troponin is released due to the lowered affinity for calcium at the binding sites (Fig. 22).
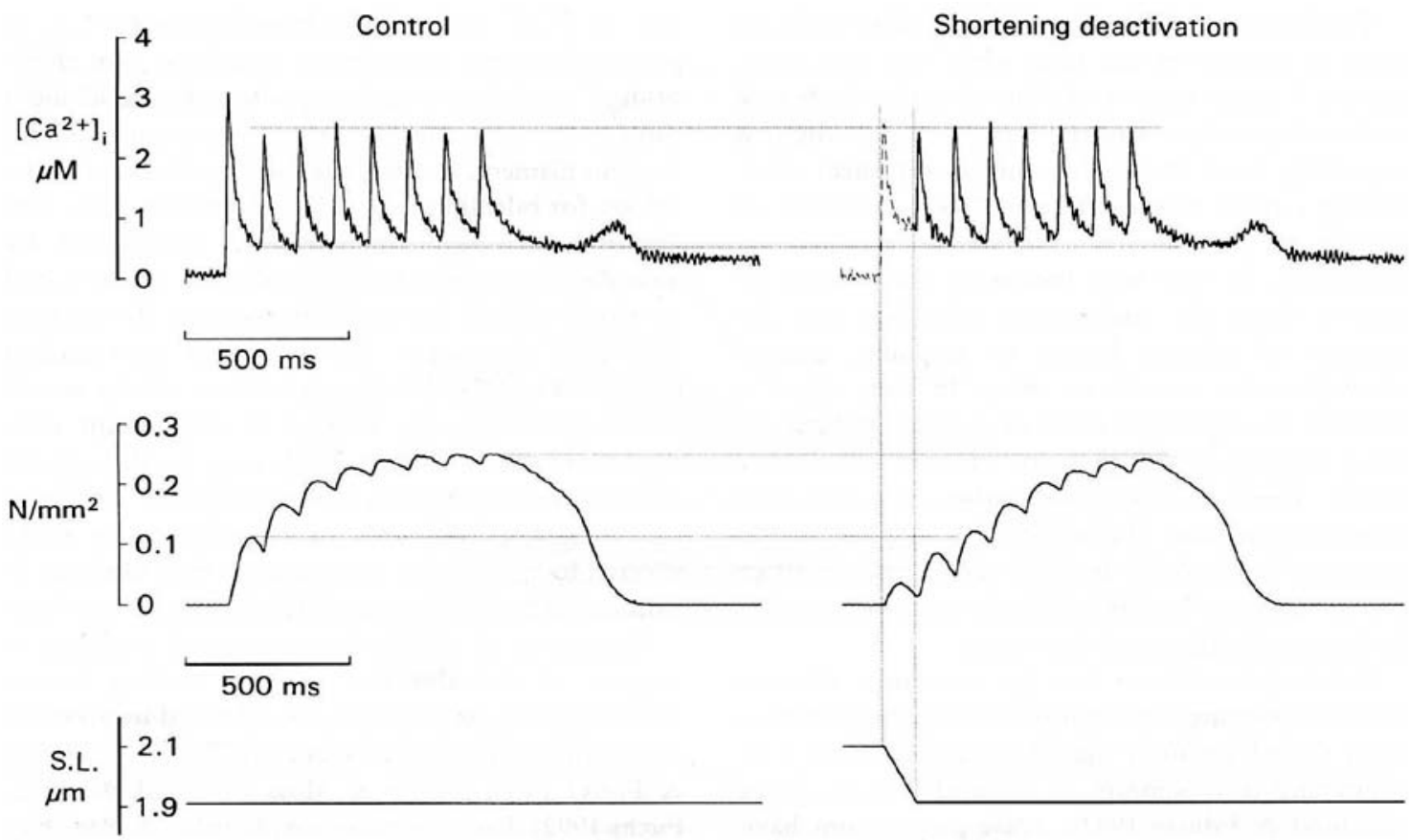

Fig. 22. Simultaneous recordings of intracellular free calcium ( $\left[\mathrm{Ca}^{2+}\right]_{\mathrm{i}}$ ) and force (middle traces) during a partially fused tetanus of a single muscle fibre of Rana temporaria. The bottom trace shows puller position calibrated in $\mu \mathrm{m}$ sarcomere length. Left panel: isometric contraction at $1.91 \mu \mathrm{m}$ sarcomere length; right panel: the contraction is initiated at $2.1 \mu \mathrm{m}$ sarcomere length and the fibre is allowed to shorten to $1.91 \mu \mathrm{m}$ during the first twitch period. The transitory force depression by shortening is quite similar to that described in Figure 18. Note that $\left[\mathrm{Ca}^{2+}\right]_{\mathrm{i}}$ is substantially higher immediately after the end of shortening as compared with the corresponding time in the control. However, from the second stimulus onwards, the $\mathrm{Ca}^{2+}{ }_{\mathrm{i}}$ transient is almost identical to that recorded in the control run, while the force remains depressed and only slowly recovers after the shortening. The approach used for the intracellular calcium measurement has been described by Caputo, Edman, Lou \& Sun, 1994.

There is reason to believe that shortening induced deactivation plays a part in normal life. Mammalian motor units are activated to produce partially fused tetani and under such conditions the movement effect may be expected to be pronounced as demonstrated in Figs. 
18 and 22. The movement effect may set a limit to what the body muscles are able to achieve, for example during running, weightlifting etc. This might in fact be the main purpose of the effect, to serve as a safety mechanism to prevent overuse of the muscles.

\section{Differences in kinetic properties along individual muscle fibres}

It is well established that individual muscles in the body vary with respect to their maximum speed of shortening Close 1972; Buchthal \& Schmalbruch, 1980). Marked differences in kinetic properties are also known to exist among individual fibres in a muscle, and there is abundant evidence to suggest that these differences in mechanical performance among muscles and individual muscle fibres are based on differences in isomyosin composition of the myofilament system (Schiaffino \& Reggiani, 1996; Bottinelli \& Reggiani, 2000). Marked

differences in shortening characteristics may exist among fibres in a given muscle. This applies in particular to mammalian and avian skeletal muscles, but substantial differences in shortening kinetics, although less pronounced than those observed among mammalian muscle fibres (Julian et al. 1981), are also observed among twitch fibres of amphibian skeletal muscles (Edman \& Hwang, 1977; Edman, 1979). 
$A$

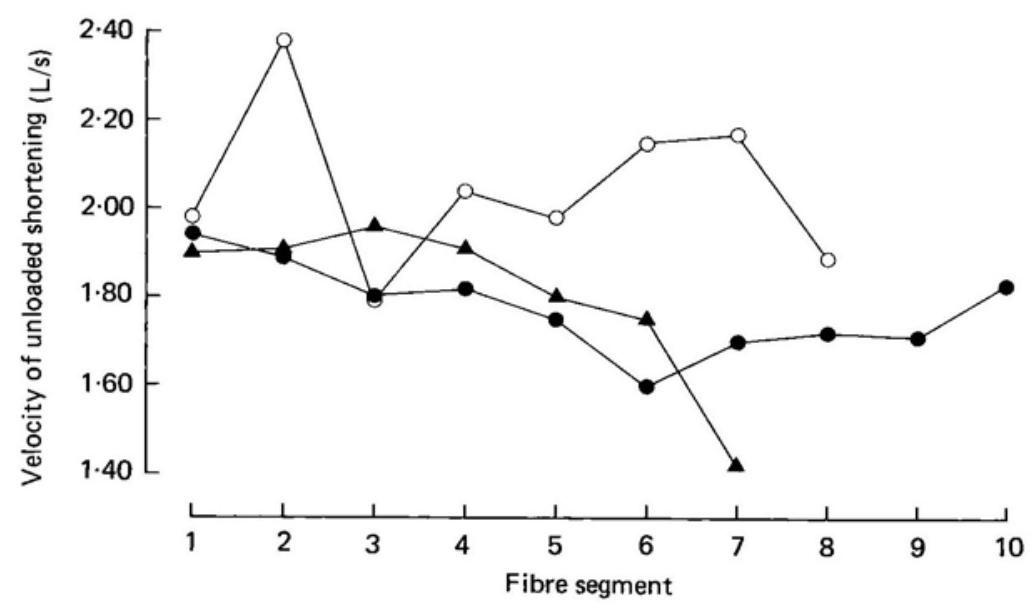

$B$

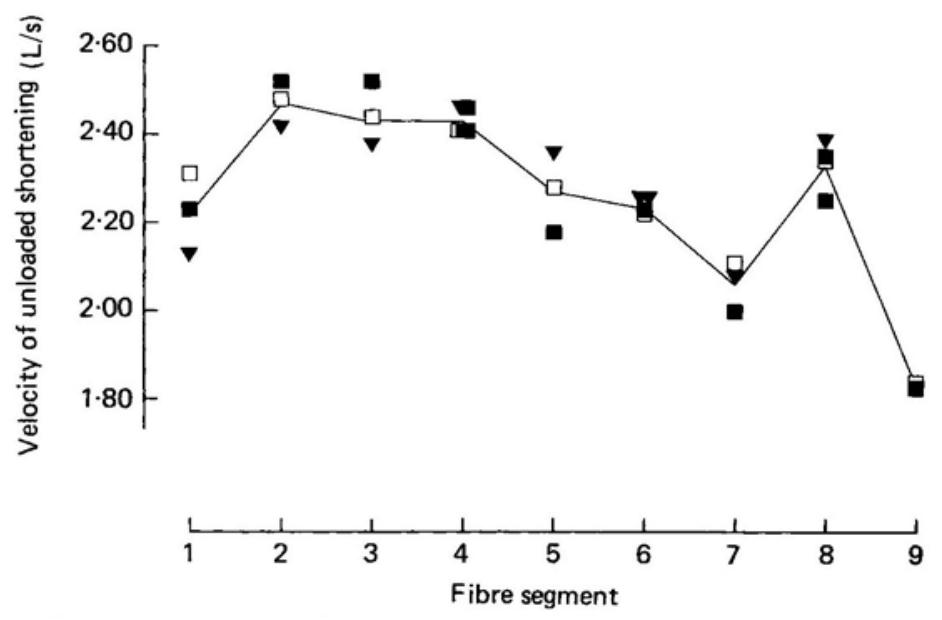

Fig. 23. A. Velocity of unloaded shortening $\left(V_{0}\right)$ recorded in consecutive segments of three different muscle fibres. Length of segments, 0.6-0.8 mm. Total fibre length at $2.25 \mu \mathrm{m}$ sarcomere length: $\circ, 7.65 \mathrm{~mm} ; \bullet, 8.40$ $\mathrm{mm} ; \boldsymbol{\Lambda}, 6.25 \mathrm{~mm}$. Note that each fibre has a unique $V_{0}$ pattern and that, in certain regions of the fibre, there is a steady increase or decrease in $V_{0}$ over several segments in succession. Data points are values of two or more recordings in each segment. $B$. Repeated $V_{0}$ measurements along a single muscle fibre during different time intervals after the onset of the experiment illustrating consistency of the measurements over several hours. Time interval after onset of experiment: $\square$, 30-65 min; $\mathbf{~ , ~ 7 0 - 1 0 5 ~ m i n ; ~ \nabla , ~ 2 0 0 - 2 3 0 ~ m i n . ~ F r o m ~ E d m a n ~ e t ~ a l . ~ ( 1 9 8 5 ) . ~}$

There is now evidence that the kinetics of shortening may even differ substantially from one part to another within individual fibres in vertebrate striated muscles. Experimental data illustrating this point are shown in Fig. 23. In these experiments (Edman et al., 1985) opaque markers were placed on the fibre surface, $0.5-0.8 \mathrm{~mm}$ apart, along the length of the preparation. The change in distance between two adjacent markers (one segment) was monitored by means of a photoelectric recording system, while the fibre was released to 
shorten isotonically against a load close to zero during tetanic stimulation. The speed of shortening $\left(\mathrm{V}_{0}\right)$ was recorded during repeated tetani from each of the segments, the accuracy of these measurements being better than $4 \%$ in all parts of the fibre. As exemplified in Fig. 23 A the maximum velocity of shortening differed substantially along the fibres, each fibre exhibiting a unique pattern of velocities. In general there was a clear trend towards an increase or decrease in $\mathrm{V}_{0}$ over a sequence of segments, but in some cases an abrupt, large change in velocity was seen between two adjacent segments. The observed differences in velocity along a given fibre remained the same (within the accuracy of the measurement) over several hours of experimentation (Fig. $23 \mathrm{~B}$ ). It is noteworthy that the segmental differences in $\mathrm{V}_{0}$ did not bear any relation to the passive elastic properties of the muscle fibres (see further Edman et al., 1985) supporting the view that the observed differences in shortening velocity do reflect true differences in the kinetic properties of the myofilament system along the fibre.

The segmental differences in $\mathrm{V}_{0}$ were found to be associated with specific differences in the shape of the force-velocity relationship. Segments having a lower $\mathrm{V}_{0}$ were consistently found to be less curved, i.e. to have a higher value of $\mathrm{a} / \mathrm{P}_{0}$ in terms of Hill's (1938) hyperbolic equation. This finding has an interesting similarity with the original (two-step) cross-bridge model of muscle contraction (Huxley, 1957) according to which an increase in $\mathrm{V}_{0}$ is predicted to be associated with an increased curvature of the force-velocity relationship. It should be noted, however, that this relation between $\mathrm{V}_{0}$ and the curvature of the force-velocity relation is opposite to what is generally found when 'fast' and 'slow' muscles are compared. This apparent disagreement is not understood at the present time and requires further studies to be explained. 


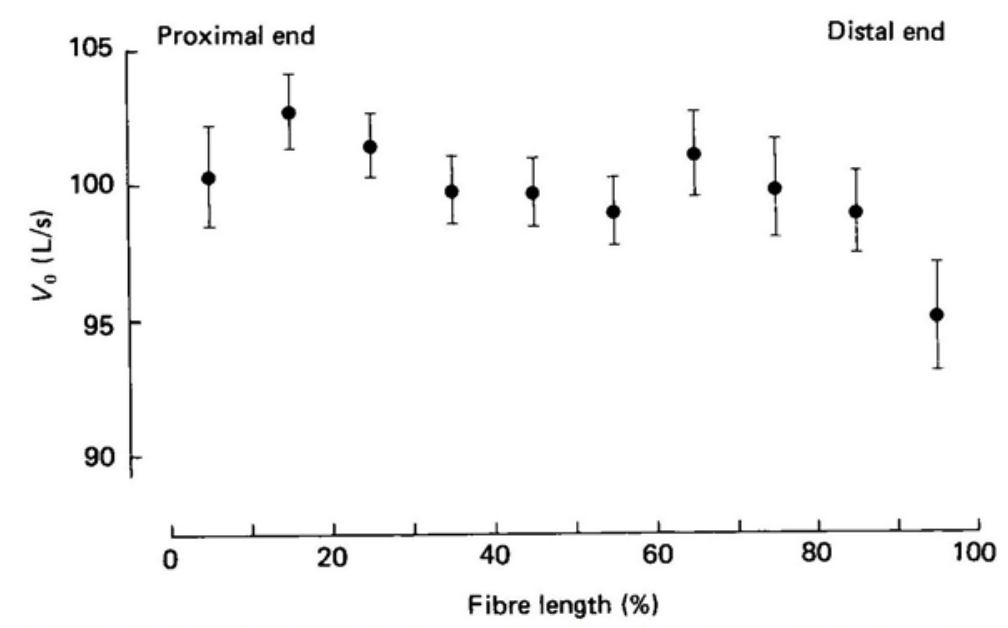

Fig. 24. $V_{0}$ of individual fibre segments related to the fibre's orientation in the body. The length of each fibre has been normalized to $100 \%$. $V_{0}$ estimated for ten successive segments of equal length from proximal (left) to distal (right) end of the fibre. Data points are mean values ( \pm S.E. of mean) based on measurements in fourteen fibres. From Edman et al. (1985).

It was realized that the segmental differences in contractile properties along individual muscle fibres might somehow be related to the fibre's orientation in the body. A series of experiments was therefore carried out in which the fibre ends were identified as 'proximal' and 'distal' with respect to the fibre's attachment between the knee and ankle of the frog's leg. Fig. 24 summarizes the results obtained from 14 fibres in which $V_{0}$ was determined in 10 successive segments in each preparation. The orientation of the fibre in the body does indeed seem to have some influence upon the $\mathrm{V}_{0}$ pattern. Thus, there was a clear trend towards higher velocities in the proximal part of the fibre and towards lower velocities in the most distal part (For details concerning the statistical treatment, see Edman et al. 1985).

A variety of isomyosins are now identified in striated muscle and their existence has been found to correlate with specific kinetic properties of individual fibres in both mammalian and amphibian muscles (e.g. Lännergren \& Hoh, 1984; Spurway \& Rowlerson , 1989; Schiaffino \& Reggiani, 1996; Bottinelli \& Reggiani, 2000). Co-existence of isomyosins of different kinetic types has been demonstrated in individual muscle fibres (Edman et al. 1988) and appears to be a common phenomenon. A non-uniform isomyosin composition along the muscle fibre may, at least partly, account for the segmental differences in $\mathrm{V}_{0}$. 
Inhomogeneities of the isomyosin pattern along the fibre do not seem unreasonable in view of the multicellular origin of the muscle fibre. A skeletal muscle fibre, which in every respect functions as a single cell, is in reality a syncytium of several myoblasts that have fused (Bloom \& Fawcett, 1975). The genetic information contained in a given fibre thus stems from multiple sources, and the genetic instruction may therefore differ along the fibre. As a result certain features of the contractile system may become more pronounced in one region than in another. However, as demonstrated in Fig. 24, the orientation of the muscle fibre in the body does seem to play a part as well. The differences in contractile performance along the fibre may therefore not be entirely accidental but somehow related to the close environment in which the fibre and the muscle operate in the body. 


\section{References}

Abbott, B.C. \& Aubert, X.M. (1952). The force exerted by active striated muscle during and after change of length. Journal of Physiology 117, 77-86.

Aubert, X. (1956). Le couplage energetique de la contraction musculaire. These d'agregation l'enseignement superieur. Editions Arsaia, Bruxelles

Bailey, K. (1937). Composition of the myosins and myogen of skeletal muscle. Biochemical Journal 31, 1406-1413.

Bárány, E.H., Edman, K.A.P. \& Palis, A. (1951a). The influence of electrolytes on the rate of viscosity drop in ATP-actomyosin mixtures. Acta Physiologica Scandinavica 24, 361367.

Bárány, E.H., Edman, K.A.P. \& Palis, A. (1952). The influence of potassium chloride concentration on the rate of drop of viscosity in ATP-actomyosin mixtures. Acta Societas Medicorum Upsaliensis 56, 269-272.

Bárány, E.H., Edman, K.A.P., Högberg, F. \& Rosner, H. (1951b). A recording viscosimeter for study of rapid viscosity changes. Acta Physiologica Scandinavica 23, 128-136.

Bárány, M. (1967). ATPase activity of myosin correlated with speed of muscle shortening. Journal of General Physiology 50, 197-218.

Blinks, J.R., Rüdel, R. \& Taylor, S.R. (1978) Calcium transients in isolated amphibian skeletal muscle fibres: detection with aequorin. Journal of Physiology 277, 291-323.

Blix, M. (1894). Die Länge und die Spannung des Muskels. Skandinavisches Archiv für Physiologie 5, 173-206.

Bloom, W. \& Fawcett, D.W. (1975). A Textbook of Histology, p. 328. Philadelphia: W.B. Saunders Company.

Bottinelli, R. \& Reggiani, C. (2000). Human skeletal muscle fibres: molecular and functional diversity. Progress in Biophysics and Molecular Biology 73, 195-262. 
Briden , K. L. \& Alpert, N. R. (1972). The effect of shortening on the time-course of active state decay. Journal of General Physiology 60, 202-220.

Brown, L.M. \& Hill, L. (1991). Some observations on variations in filament overlap in tetanized muscle fibres and fibres stretched during a tetanus, detected in the electron microscope after rapid fixation. Journal of Muscle Research and Cell Motility 12, 171182.

Buchthal, F. \& Schmalbruch, H. (1980). Motor unit of mammalian muscle. Physiological Reviews 60, 90-142.

Caputo, C., Edman, K.A.P., Lou, F. \& Sun, Y.-B. (1994). Variation in myoplasmic $\mathrm{Ca}^{2+}$ concentration during contraction and relaxation studied by the indicator flyo-3 in frog muscle fibres. Journal of Physiology 478, 137-148.

Cavagna, G.A. \& Citterio, G. (1974). Effect of stretching on the elastic characteristics and the contractile component of frog striated muscle. Journal of Physiology 239, 1-14.

Close, R. (1972). Dynamic properties of mammalian skeletal muscles. Physiological Reviews 52, 129-197.

Devrome, A.N.\& MacIntosh, B.R. (2007). The biphasic force-velocity relationship in whole rat skeletal muscle in situ. Journal of Applied Physiology 102, 2294-2300.

Duke, T.A. (1999). Molecular model of muscle contraction. Proceedings of the National Academy of Sciences U.S.A. 96, 2770-2775.

Edman, K.A.P. \& Andersson, K.-E. (1968). The variation in active tension with sarcomere length in vertebrate skeletal muscle and its relation to fibre width. Experientia 24, 134136.

Edman, K.A.P. \& Hwang, J.C. (1977). The force-velocity relationship in vertebrate muscle fibres at varied tonicity of the extracellular medium. Journal of Physiology 269, 255272. 
Edman, K.A.P. \& Kiessling, A. (1971). The time course of the active state in relation to sarcomere length and movement studied in single skeletal muscle fibres of the frog. Acta Physiologica Scandinavica 81, 182-196.

Edman, K.A.P. \& Lou, F. (1992). Myofibrillar fatigue versus failure of activation during repetitive stimulation of frog muscle fibres. Journal of Physiology 457, 655-673.

Edman, K.A.P. \& Nilsson, E. (1968). The mechanical parameters of myocardial contraction studied at a constant length of the contractile element. Acta Physiologica Scandinavica 72, 205-219.

Edman, K.A.P. \& Nilsson, E. (1972). Relationship between between force and velocity of shortening in rabbit papillary muscle. Acta Physiologica Scandinavica 85, 488-500.

Edman K.A.P. \& Reggiani, C. (1984). Redistribution of sarcomere length during isometric contraction of frog muscle fibres and its relation to tension creep. Journal of Physiology 351, 169-198.

Edman, K.A.P. \& Reggiani, C. (1987). The sarcomere length-tension relation determined in short segments of intact muscle fibres of the frog. Journal of Physiology 385, 709-732.

Edman, K.A.P. \& Schild, H.O. (1962). The need for calcium in the contractile responses induced by acetylcholine and potassium in rat uterus. Journal of Physiology 161, 424441.

Edman, K.A.P. \& Schild, H.O. (1963). Calcium and the stimuland and inhibitory effects of adrenaline in depolarized smooth muscle. Journal of Physiology 169, 404-411.

Edman, K.A.P. \& Tsuchiya, T. (1996). Strain of passive elements during force enhancement by stretch in frog muscle fibres. Journal of Physiology 490, 191-205.

Edman, K.A.P. (1950). The action of ouabain on heart actomyosin. Acta Physiologica Scandinavica 21, 230-237. 
Edman, K.A.P. (1951). The action of ouabain on actomyosin from striated musculature. Acta Physiologica Scandinavica 23, 137-142.

Edman, K.A.P. (1953). Action of cardiac glycosides on the ATP-induced contraction of glycerinated muscle fibers. Acta Physiologica Scandinavica 30, 69-79.

Edman, K.A.P. (1959a). Relaxation of glycerol-extracted muscle fibre bundles induced by zinc in the presence of ATP and other polyphosphates. Acta Physiologica Scandinavica 46, 62-87.

Edman, K.A.P. (1959b). The binding of zinc to glycerol-extracted muscle, and its relaxing effect. Acta Physiologica Scandinavica 46, 209-227.

Edman, K.A.P. (1966). The relation between sarcomere length and active tension in isolated semitendinosus fibres of the frog. Journal of Physiology 183, 407-417.

Edman, K.A.P. (1975). Mechanical deactivation induced by active shortening in isolated muscle fibres of the frog. Journal of Physiology 246, 255-275.

Edman, K.A.P. (1979). The velocity of unloaded shortening and its relation to sarcomere length and isometric force in vertebrate muscle fibres. Journal of Physiology 291, 143159.

Edman, K.A.P. (1980). Depression of mechanical performance by active shortening during twitch and tetanus of vertebrate muscle fibres. Acta Physiologica Scandinavica 109, 15 26.

Edman, K.A.P. (1981). Deactivation of the contractile system induced by shortening of striated muscle. In The Regulation of Muscle Contraction: Excitation-contraction coupling. Eds.: Grinnell \& Brazier. Academic Press, 1981. pp. 281-296.

Edman, K.A.P. (1988). Double-hyperbolic force-velocity relation in frog muscle fibres. Journal of Physiology 404, 301-321. 
Edman, K.A.P. (1999). The force bearing capacity of frog muscle fibres during stretch: its relation to sarcomere length and fibre width. Journal of Physiology 519, 515-526.

Edman, K.A.P. (2005). Contractile properties of mouse single muscle fibers, a comparison with amphibian muscle fibers. Journal of Experimental Biology 208, 1905-1913.

Edman, K.A.P., Caputo, C. \& Lou, F. (1993). Depression of tetanic force induced by loaded shortening of frog muscle fibres. Journal of Physiology 446, 535-552.

Edman, K.A.P., Elzinga, G. \& Noble, M.I.M. (1978). Enhancement of mechanical performance by stretch during tetanic contractions of vertebrate skeletal muscle fibres. Journal of Physiology 281, 139-155.

Edman, K.A.P., Elzinga, G. \& Noble, M.I.M. (1982). Residual force enhancement after stretch of contracting frog single muscle fibers. Journal of General Physiology 80, 769784.

Edman, K.A.P., Månsson, A. \& Caputo, C. (1997). The biphasic force-velocity relationship in frog muscle fibres and its evaluation in terms of cross-bridge function. Journal of Physiology 503, 141-156.

Edman, K.A.P., Radzyukevich \& Kronborg, B. (2002). Contractile properties of isolated muscle spindles of the frog. Journal of Physiology 541, 905-916.

Edman, K.A.P., Reggiani, C. \& te Kronnie, G. (1985). Differences in maximum velocity of shortening along single muscle fibres of the frog. Journal of Physiology 365, 147-163.

Edman, K.A.P., Reggiani, C., Schiaffino \& te Kronnie, G. (1988). Maximum velocity of shortening related to myosin isoform composition in frog skeletal muscle fibres. Journal of Physiology 395, 679-694.

Ekelund, M.C. \& Edman, K.A.P. (1982). Shortening induced deactivation of skinned fibres of frog and mouse striated muscle. Acta Physiologica Scandinavica. 116. 189-199.

Elliott, A. \& Offer, G. (1978). Shape and flexibility of the myosin molecule. Journal of Molecular Biology 123, 505-519. 
Elliott, G.F., Lowy, J. \& Worthington, C.R. (1963). An X-ray and light-diffraction study of the filament lattice of striated muscle in the living state and in rigor. Journal of Molecular Biology 6, 295-305.

Engelhardt, W. A: \& Ljubimowa, M. N. (1939). Myosine and Adenosinetriphosphatase. Nature 144, 668-669.

Evans, C.L. \& Hill, A.V. (1914). The relation of length to tension development and heat production on contraction in muscle. Journal of Physiology 49, 10-16.

Fenn, , W.O. \& Marsh, B.S. (1935). Muscular force at different speeds of shortening. Journal of Physiology 85, 277-297.

Fenn, W.O. (1924). The relationship between work performed and the energy liberated in muscular contraction. Journal of Physiology 58, 373-395.

Gordon, A.M., Huxley, A.F. \& Julian, F.J. (1966). The variation in isometric tension with sarcomere length in vertebrate muscle fibres. Journal of Physiology 184, 170-192.

Granzier, H.L. \& Pollack, G.H. (1989). Effect of active pre-shortening on isometric and isotonic performance of single frog muscle fibres. Journal of Physiolohy 415, 299-327.

Herzog, W., Lee, E. J. \& Rassier, D. E. (2006). Residual force enhancement in skeletal muscle. Journal of Physiology 574, 635-642.

Hill, A.V. \& Howarth, J.V. (1959). The reversal of chemical reactions in contracting muscle during an applied stretch. Proceedings of the Royal Society B, 151, 169-193.

Hill, A.V. (1938). The heat of shortening and the dynamic constants of muscle. Proceedings of the Royal Society B 126, 136-195.

Huxley, A.F. \& Niedergerke, R. (1954). Structural changes in muscle during contraction: Interference microscopy of living muscle fibres. Nature 173, 971-973.

Huxley, H.E. \& Hanson, J. (1954). Changes in the cross-striations of muscle during contraction and stretch and their natural interpretation. Nature 173, 973-977. 
Huxley, H.E. (1969). The mechanism of muscular contraction. Science 164, 1356-1366.

Huxley, H.E. (1973). Molecular basis of contraction in cross-striated muscles. In The

Structure and Function of Muscle, vol. 1, $2^{\text {nd }}$ edn., ed Bourne, G., pp. 301-387. New York: Academic Press.

Jewell, B. R. \& Wilkie D. R. (1960). The mechanical properties of relaxing muscle. Journal of Physiology 152, 30-47.

Joumaa, V, Leonard, T.R. \& Herzog, W. (2008). Residual force enhancement in myofibrils and sarcomeres. Proceedings: Biological Sciences 275, 1411-1419.

Joyce, G.C., Rack, P.M.H., Westbury, D.R. (1969). The mechanical properties of cat soleus muscle during controlled lenghthening and shortening movements. Journal of Physiology 204, 461-474.

Julian , F.J. \& Morgan, D.L. (1979). The effect on tension of non-uniform distribution of length changes applied to frog muscle fibres. Journal of Physiology 293, 379-392.

Julian, F.J., Moss, R.L.. \& Waller, G.S. (1981). Mechanical properties and myosin light chain composition of skinned muscle fibres from adult and new-born rabbits. Journal of Physiology 311, 201-218.

Katz, B. (1939). The relation between force and speed in muscular contraction. Journal of Physiology 96, 54-64.

Lännergren, J. \& Hoh, J.F.Y. (1984). Myosin isoenzymes in single muscle fibres of Xenopus laevis: analysis of five different functional types. Proceedings of the Royal Society B 222, 401-408.

Lee, E.J. \& Herzog, W. (2008). Residual force enhancement exceeds the isometric force at optimal sarcomere length for optimized stretch conditions. Journal of Applied Physiology 105, 457-462.

Lou, F. \& Sun, Y.-B. (1993). The high-force region of the force-velocity relation in frog skinned muscle fibres. Acta Physiologica Scandinavica 148, 243-252. 
Maréchal, G. \& Plaghki, L. (1979). The deficit of the isometric tetanic tension redeveloped after a release of frog muscle at constant velocity. Journal of General Physiology 73, 453-467.

Millman, B. M. (1998). The filament lattice of striated muscle. Physiological Reviews 78, 359-391.

Mitsui, T. \& Chiba, H. (1996). Proposed modification of the Huxley-Simmons model for myosin head motion along an actin filament. Journal of Theoretical Biology 182, 147159.

Nielsen, B.G. (2003). Unfolding transitions in myosin give rise to the double hyperbolic force-velocity relation in muscle. Journal of Physics: Condensed Matter 15, 1759-1765.

Page, S.G. (1968). Fine structure of tortoise skeletal muscle. Journal of Physiology 197, 709-715..

Ralston, H.J., Inman, V.T., Strait, L.A. \& Shaffrath, M.D. (1947). Mechanics of human isolated voluntary muscle. American Journal of Physiology 151, 612-620.

Ramsey, R.W. \& Street, S.F. (1940). The isometric length tensiondiagram of isolated skeletal muscle fibres of the frog. Journal of Cellular and Comparative Physiology 14, 11-34.

Rassier, D. E., Herzog, W. \& Pollack, G. H. (2003). Dynamics of individual sarcomeres during and after stretch in activated single myofibrils. Proceedings: Biological Sciences $270,1735-1740$.

Rassier, D.E. \& Herzog, W. (2004a). Effects of shortening on stretch-induced force enhancement in single skeletal muscle fibers. Journal of Biomechanics 37, 1305-1312.

Rassier, D.E. \& Herzog, W. (2004b). Considerations on the history dependence of muscle contraction. Journal of Applied Physiology 96, 419-427. 
Rayment, I., Rypniewsky, W.R., Schmidt-Bäse, K., Smith, R., Tomchick, D.R., Benning, M.M., Winkelmann, D.A., Wesenberg, G. \& Holden, H.M. (1993). Three-dimensional structure of myosin subfragment-1: A molecular motor. Science 261, 50-58.

Reggiani, C. (2007). When fibres go slack and cross bridges are free to run: a brilliant method to study kinetic properties of acto-myosin interaction. Journal of Physiology 583, 5-7.

Schachar, R., Herzog, W. \& Leonard, T.R. (2004). The effects of muscle stretching and shortening on isometric forces on the descending limb of the force-length relationship. Journal of Biomechanics 37, 917-926.

Schiaffino, S. \& Reggiani, C. (1996). ). Molecular diversity of myofibrillar proteins: gene regulation and functional significance. Physiological Reviews 76, 371-423.

Sosa, H., Popp, D., Ouyang, G. \& Huxley, H.E. (1994). Ultrastructure of skeletal muscle fibers studied by a plunge quick freezing method: myofilament lengths. Biophysical Journal 67, 283-292.

Spurway, N.C. \& Rowlerson, A.M. (1989). Quantitative analysis of histochemical and immunohistochemical reactions in skeletal muscle fibres of Rana and Xenopus.

Histochemical Journal 21, 461-471.

Straub, F.B. (1942). Actin. Studies from the Institute of Medical Chemistry University Szeged, 2, 3-15.

Sugi, H. \& Tsuchiya, T. (1988). Stiffness changes during enhancement and deficit of isometric force by slow length changes in frog skeletal muscle fibres. Journal of Physiology 407, 215-229.

Sugi, H. (1972). Tension changes during and after stretch in frog muscle fibers. Journal of Physiology 225, 237-253.

Szent-Györgyi, A. (1945). Studies on muscle. Acta Physiologica Scandinavica 9, suppl. 25, $1-128$. 
Talbot, J. A. \& Morgan, D. L. (1996). Quantitative analysis of sarcomere non-uniformities in active muscle following a stretch. Journal of Muscle Research and Cell Motility 17, 261-268.

Telley, I. A., Stehle, R., Ranatunga, K.W., Pfitzer, G., Stüssi, E. \& Denoth, J. (2006). Dynamic behaviour of half-sarcomeres during and after stretch in activated rabbit psoas myofibrils: sarcomere asymmetry but no 'sarcomere popping'. Journal of Physiology $573,173-185$.

Weber, H.H. \& Portzehl, H. (1952). Kontraktion, ATP-Cyclus und fibrilläre Proteine des Muskels. Ergebnisse der Physiologie 47, 369-468. 